\title{
Der Aufsichtsrat
}

\section{- Bedeutungswandel, Konvergenz, unternehmerische Mitverantwortung, Pflichten- und Haftungszuwachs -}

\author{
Professor Dr. Dr. Klaus J. Hopt, Hamburg*
}

\section{Inhaltsübersicht}

ZGR 2019, 507-543

I. Zur Entwicklungsgeschichte des Aufsichtsrats . . . . . . . . . . . . . . . . 508

1. Die Anfänge: Verwaltungsrat und Aufsichtsrat . . . . . . . . . . . . . . . . 508

2. Der Aufsichtsrat im rheinischen Kapitalismus . . . . . . . . . . . . . . . 511

3. Unternehmerische Mitbestimmung, Rückzug der Banken aus den Aufsichtsräten großer Gesellschaften und Aufsichtsratsreformen seit den 1990 er Jahren . . . . . . . . . . . . . . . . . . . .

II. Konvergenz des ein- und des zweistufigen Systems bei verbleibenden Pfad-

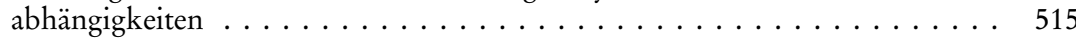

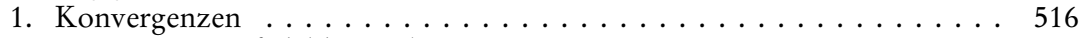

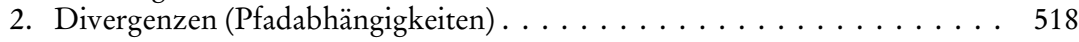

III. Die unternehmerische Mitentscheidung: Aufgaben des Aufsichtsrats zusammen mit dem Vorstand und allein . . . . . . . . . . . . . . .

1. Prospektive Überwachung und unternehmerische Entscheidungen des

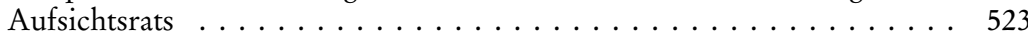

2. Aufsichtsrat und Information . . . . . . . . . . . . . . . . . . . 527

3. Aufsichtsrat und Interessenkonflikte . . . . . . . . . . . . . . 531

IV. Der zu zahlende Preis: Mehr Pflichten und strengere zivil- und strafrechtliche Haftung von Aufsichtsratsmitgliedern . . . . . . . . . . . . 538

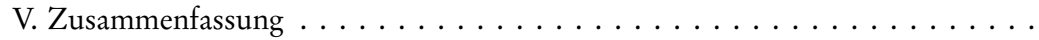

Der vorliegende Beitrag nimmt die Neukommentierungen des Aufsichtsratsrechts 2019 zum Anlass eines Rückblicks und Ausblicks zum Aufsichtsrat. Der Aufsichtsrat hat seit den Neunziger Jabren durch Reformen in Diskussion und Praxis ganz erbeblich an Bedeutung gewonnen. Er kann sich auch international nicht nur ohne weiteres gegenüber dem Verwaltungsrat (board) behaupten, sondern es lässt sich insgesamt sogar eine deutliche Konvergenz beider Systeme feststellen. Der Auf-

* Direktor em. und Professor am Max Planck Institut für ausländisches und internationales Privatrecht Hamburg. Der Aufsatz geht auf einen Vortrag auf dem Geburtstagssymposion für Peter Doralt, am 8.4.2019 an der WU Wien zurück und ist diesem gewidmet, Organisatoren waren seine Schüler Susanne Kalss und Martin Winner. Diese hatten im Hinblick auf den Jubilar um einen Vortrag mit dem Thema „Die unglaubliche Karriere des Aufsichtsrats“ gebeten. Zu Person und Wirken Peter Doralts Kalss, in: Grundmann/Riesenhuber, Deutschsprachige Zivilrechtslehrer des 20. Jahrhunderts in Berichten ihrer Schüler, 2010, S. 185. Englischsprachige Ausgabe: KaLss, in: Grundmann/Riesenhuber, Private Law Development in Context, German Private Law and Scholarship in the 20th Century, 2018, p. 537. 
sichtsrat ist inzwischen zu einem mitunternehmerischen Organ, wenn auch keinem Geschäftsführungsorgan, geworden. Das wird de lege lata an drei Problemfeldern aufgezeigt: prospektive Überwachung und unternehmerische Entscheidungen des Aufsichtsrats, Aufsichtsrat und Information und Aufsichtsrat und Interessenkonflikte. Die durchaus umstrittene Weiterentwicklung des Aufsichtsratsrechts in allen drei Problemfeldern wird am Investorendialog des Aufsichtsrats, der vorstandsunabhängigen Information und der Diskussion um die related party transactions aufgezeigt. Der Bedeutungszuwachs hat seinen Preis in mehr Pflichten und einer strengeren zivilrechtlichen und-problematischer - strafrechtlichen Haftung der Aufsichtsratsmitglieder.

2019 witnessed the publication of several new editions of legal commentaries on the law governing German supervisory boards. This gives occasion to reflect back at the rise of the supervisory board in Europe and to look forward to its future. Since the 1990s, the supervisory board has in the wake of far-reaching reforms undeniably grown in importance, both in theory and practice. One can even identify a clear convergence of the one-tier and the two-tier systems. The supervisory board has become a co-entrepreneurial organ of stock corporations, even if not a true management organ. This can be demonstrated by the developments in three fields: prospective control and business decisions of the supervisory board, the supervisory board and information, and the supervisory board and conflicts of interest. This article will analyze these developments through the lens of three very controversial problems: the dialogue between investors and the supervisory board, the right of the supervisory board to obtain information directly from employees, and the reform of the law governing related party transactions. The increase in importance of the supervisory board also carries certain costs - namely, broader legal duties and a more exacting liability of its members, both under civil and criminal law.

\section{Zur Entwicklungsgeschichte des Aufsichtsrats}

\section{Die Anfänge: Verwaltungsrat und Aufsichtsrat}

Der Aufsichtsrat steht heute mitten in der rechtswissenschaftlichen und politischen Diskussion. Gerade sind drei große Kommentierungen des Aufsichtsratsrechts der Aktiengesellschaft erschienen, ${ }^{1}$ die Umsetzung der Aktionärs-

1 Норт/Roth, Großkomm. z. AktG, Bd. 5, 5. Aufl., 2019, \$S 95-116, auch: Hopt/Roth, Der Aufsichtsrat: Aktienrecht und Corporate Governance, Sonderausgabe aus dem Großkomm. z. AktG $\$ \mathbb{S} 95$ bis 116, 2019; dazu Hорт/Roth, Großkomm. z. AktG, Bd. 4/2, 5. Aufl., 2015, $\$ 93$; Habersack, Münchener Komm. z. AktG, Bd. 2, 5. Aufl., 2019, S\$ 95-116, mit Angaben zum österreichischen Aufsichtsratsrecht durch KaLss; SpINDLER, in: Spindler/Stilz, Komm. z. AktG, 4. Aufl., 2019, \S 95-116; auch KocH, in: Hüffer/Koch, Komm. z. AktG, 13. Aufl., 2018. Auf Englisch Hорт, The German Law of and Experience with the Supervisory Board, in: Veil/Gao, Foreign Investments on Chinese Capital Markets, 2017, S. 121. Zum österreichischen Aufsichtsrat Kalss, in: Doralt/ Nowotny/Kalss, Komm. z. AktG, 2. Aufl., 2012, \$S 86-99. Zum schweizerischen Verwaltungsrat Böckli, Schweizer Aktienrecht, 4. Aufl., 2009, \$13; Meier-Hayoz/ForstMOSER/SETHE, Schweizerisches Gesellschaftsrecht, 12. Aufl., 2018, OR $707 \mathrm{ff} \ 16 \mathrm{~N}$. 568 ff; Nobel, Berner Kommentar, 2017 \ 9 N. 102 ff. 
rechte-Richtlinie durch das ARUG II(-E) zu den related party transactions ${ }^{2}$ führt zu noch mehr Bedeutung des Aufsichtsrats, und die Reform des Deutschen Corporate Governance Kodex mit wichtigen Aufgaben für den Aufsichtsrats steht für Ende des Jahres an ${ }^{3}$. Um diesen Aufschwung und die heutige Bedeutung des Aufsichtsrats einschätzen zu können, soll das Thema vierfach beleuchtet werden: entwicklungsgeschichtlich, rechtsvergleichend, rechtsdogmatisch und abschließend rechtspraktisch mit Blick auf den Preis, den die Aufsichtsratsmitglieder für diesen Bedeutungszuwachs zu zahlen haben. Zunächst ist ein kurzer Blick auf seinen Beginn in der ersten Hälfte des

2 1) BMJV, RefE ARUG II v. 11.10.2018, \\ 107 Abs. 4, 111a, 111b, 111c; dazu unter vielen Anzinger, ZGR 2019, 39; Bayer, DB 2018, 3034; Bayer/J. Schmidt, BB 2018, 2562, $2572 \mathrm{ff}$; Bungert/Berger, DB 2018, 2801, 2868; DAV Handelsrechtsausschuss, NZG 2019, 12; Engert/Florstedt, ZIP 2019, 493, 503; Gesellschaftsrechtliche VereiniGUNG/VGR, AG 2018, 920; Habersack/Verse, Europäisches Gesellschaftsrecht, 5. Aufl., 2019, $\$ 7$ Rdn. 54 ff, 80; Heldt, AG 2018, 905; Kleinert/Mayer, EuZW 2019, 103; LieDer/Wernert, ZIP 2018, 2441; Müller, ZGR 2019, 97; Orth/Oser/Philippsen/Sultana, DB 2019, 230; Pälicke, Der Konzern 2018, 369; Paschos/Goslar, AG 2018, 857, 866; J. Schmidt, NZG 2018, 1201, 1208 ff; Seidel, AG 2018, 423; Seulen, DB 2018, 2915; TrÖGer/Roth/STrenger, BB 2018, 2946; Zwirner, DB 2018, 3007.

2) RegE ARUG II v. 20.3.2019; dazu J. Schmidt, EuZW 2019, 261; Bungert/Wansleben, BB 2019, 1026; Lieder/Wernert, ZIP 2019, 989; Löbbe/Fischbach, AG 2019, 373; PAschos/Goslar, AG 2019, 365; TARdE, NZG 2019, 488; allgemeiner zum RegE WentZ, WM 2019, 906.

3) Zur Umsetzung in Österreich Diregger, GesRZ 2019, 57: für Zustimmung des Aufsichtsrats, nicht der Hauptversammlung. Weitergehende Reformvorschläge bei Kalss/ SchaUer, Die Reform des Österreichischen Kapitalgesellschaftsrechts, 16. Österreichischer Juristentag 2006, Bd. II/1, S. $383 \mathrm{ff}, 772$. Zur Umsetzung gibt es jetzt einen Ministerialentwurf, aber wegen der Regierungskrise soll es demnächst einen Entwurf aus dem Parlament geben.

Aus der Schweiz ist inoffiziell zu hören, dass die Umsetzung der EU-Regelung über die related party transactions dort im Rahmen der hängigen Aktienrechtsrevision nie ein ernsthaft diskutiertes Thema war, weder anlässlich der Vorarbeiten (Vorentwurf) noch anlässlich der bisherigen parlamentarischen Beratung (RK-N, N, RK-S). In der Vernehmlassung zum Vorentwurf 2014 wurde die Übernahme der EU-Regeln höchstens von vereinzelten Teilnehmerinnen und Teilnehmern gefordert. Auch Böckli, aaO (Fn. 1), \13 Rdn. $601 \mathrm{ff}$ : Genehmigung durch nicht betroffene Verwaltungsräte oder subsidiär durch die Generalversammlung oder materielle Angemessenheit.

4) Rechtsökonomisch und rechtsvergleichend Enriques/Tröger, eds., The Law and Finance of Related Party Transactions, Cambridge (forthcoming); EnRiQues/Hertig/KanDA/Pargendler, in: Kraakman et al., The Anatomy of Corporate Law, 3 d ed., 2017, ch. 6, p. 145 et seq. Auch OECD, Related Party Transactions and Minority Shareholder Rights, 2012.

3 Regierungskommission Deutscher Corporate Governance Kodex, DCGK-E, 25.10.2018, auch abgedruckt in Норт/Rотн (Sonderausgabe), aаO (Fn. 1), S. 1807 ff; dazu 111 Stellungnahmen im Rahmen der Konsultation vom 6.11.2018 - 31.1.2019, auf der homepage der Regierungskommission, Stand: 1.2.2019. 
19. Jahrhundert und später angezeigt. ${ }^{4}$ Vor 1800 gab es in Preußen nur ganz wenige Aktiengesellschaften, erst in den dreißiger Jahren mit dem Aufkommen der Eisenbahnen begann eine Gründungswelle. ${ }^{5}$ Das erste allgemeine Aktiengesetz und Ausgangspunkt aller späterer Aktienrechtskodifikationen war der französische Code de Commerce von 1807. ${ }^{6}$ Ein als Aufsichtsrat bezeichnetes Gesellschaftsorgan - im französischen Kommanditaktiengesellschaftsgesetz von 1854 als conseil de surveillance benannt und dort zwingend - gab es erst in den 1850er Jahren. Neben die Gründer und Hauptaktionäre trat eine zunehmende Anzahl kleinerer Aktionären, die eines Schutzes bedurften. In der damaligen Statutarpraxis wurde dieses Organ als Verwaltungsrat, Aufsichtsrat und ähnlich bezeichnet, es hatte außer der Überwachungsfunktion auch eine Mitwirkungsfunktion. Das liegt an seiner Herkunft aus den Niederlanden, wo in den Handelskompagnien des 18. Jahrhunderts die Hauptpartizipanten in diesem Organ dem Vorstand zur Seite standen, ihn unterstützten und kontrollierten.?

Erst mit der Abschaffung des Konzessionssystems ${ }^{8} 1870$ wurde der Aufsichtsrat neben dem Vorstand ein zwingendes Organ. Die Vorstellung, dass eine Teilung der Verwaltung sinnvoll sei, hatte es allerdings schon vorher gegeben. Sie war in einer personellen Inkompatibilität angelegt, von der es allerdings für kleinere Aktiengesellschaften eine Ausnahme gab. ${ }^{9}$ Die zwingende Zweiteilung hatte ihren Grund darin, dass der Staat sich mit dem Normativsystem von seiner bisher mehr oder weniger eingehend ausgeübten Gründungs- und Ver-

4 Zum Folgenden Норт, Ideelle und wirtschaftliche Grundlagen der Aktien-, Bank- und Börsenrechtsentwicklung im 19. Jahrhundert, in: Coing/Wilhelm, Wissenschaft und Kodifikation des Privatrechts im 19. Jahrhundert, Bd. V, 1980, S. 128; Lieder, Der Aufsichtsrat im Wandel der Zeit, 2006, S. 65 ff; BAYer/Habersack, Aktienrecht im Wandel, Bd. I, 2007. Nunmehr umfassend Fleckner, Im Anfang war die Actie, Diss. phil. (Geschichtswissenschaften) Humboldt-Universität, 2019 (im Druck). Zu Österreich Kalss/Burger/ ECKERT, Die Entwicklung des österreichischen Aktienrechts, 2003. Zur Schweiz BüHLER, Geschichte des Aktienrechts in der Schweiz 1863-1991, 2019, S. 103 ff: Die Verwaltung.

5 Umfassende Auflistung aller preußischen Aktiengesellschaften von 1770 bis 1843 (1850) bei Fleckner, aaO (Fn. 4), S. 46 ff. Die erste Aktiengründung in Österreich war die Wiener Commerzial-, Leih- und Wechselbank (später Schwarzenbergbank) 1787, 1792, Kalss/Burger/Eckert, aaO (Fn. 4), S. 50, dort auch zu den späteren Gründungen.

6 Deutsch, in: Bayer/Habersack, Aktienrecht im Wandel, Bd. I, 2007, S. 46.

7 Dazu Gepken-Jager/van Solinge/Timmerman, eds., VOC 1602-2002, 400 Years of Company Law, Deventer 2005; darin zu den Board Models in Europe Hopt/Leyens, p. 281, und schon DIEs., ECFR 2004, 135.

8 Zur mangelnden Trennschärfe der Octroi-, Konzessions- und Normativsysteme FlecKNER, aaO (Fn. 4), S. 112 ff, 116.

9 Nach BöcKLI, Konvergenz: Annäherung des monistischen und des dualistischen Führungs- und Aufsichtssystems, in: Hommelhoff/Hopt/v. Werder, Handbuch Corporate Governance, 2. Aufl., 2009, S. 255, 256, soll dieser Gedanke der Trennung auf das römische Recht mit „gestio“ und „electio et custodia“ zurückgehen. 
haltenskontrolle über die Aktiengesellschaft zurückzog und diese einem separaten Organ, dem Aufsichtsrat, übertrug. Das war in anderen Ländern wie etwa England anders. Die frühere staatliche Kontrolle wurde dort mit den Gladstone'schen Reformen durch eine institutionalisierte Publizität bewerkstelligt, eines eigenen Kontrollorgans statt des Staats neben dem board bedurfte es danach nicht. Die Kontrolle blieb dort bis zum Ende des 19. Jahrhunderts Sache der Aktionäre. ${ }^{10}$ Die im angelsächsischen Raum und weithin in den Wirtschaftswissenschaften herrschende Vorstellung von dem primären agency conflict (nur) zwischen dem board und den shareholders geht letztlich darauf zurück.

Auch nach der zwingenden Zweiteilung der Organe, die ab 1900 zur viel diskutierten „Aufsichtsratsfrage “ führte, ${ }^{11}$ blieb es jedoch in den nächsten $50 \mathrm{Jah}-$ ren gesetzlich erlaubt und übliche Praxis, dass dem Aufsichtsrat außer der Überwachung weitere Aufgaben übertragen wurden. Die Satzung sah häufig sogar vor, dass der Aufsichtsrat dem Vorstand Weisungen erteilen konnte, die dieser befolgen musste. Erst durch das Aktiengesetz 1937 wurde die Geschäftsführung durch den Aufsichtsrat verboten, was dann in das Aktiengesetz 1965 eingegangen ist. $^{12}$

\section{Der Aufsichtsrat im rheinischen Kapitalismus}

Das Verbot der Geschäftsführung durch den Aufsichtsrat wurde schon im Gesetz nicht derart befolgt, dass dem Aufsichtsrat jedwede Einmischung in die Geschäftsführung verboten worden wäre. Schon von Gesetzes wegen hatte und hat der Aufsichtsrat wichtige mitunternehmerische Aufgaben, zu denen schon seit jeher die Bestellung von Vorstandsmitgliedern und die Ernennung des Vorstandsvorsitzenden und seines Vertreters und die Beratung gehören. In

10 Davies/Worthington, in: Gower/Davies/Worthington, Principles of Modern Company Law, $10^{\text {th }}$ ed., 2016, 14-6.

11 Lieder, aaO (Fn. 4), S. 203 ff: „papierene Sündflut“. In Österreich wurde der Aufsichtsrat erst mit der Übernahme des deutschen Aktiengesetzes 1937 im Jahre 1938 zwingend, unter dem öAHGB gab es verschiedene Organisationsmodelle je nach Satzung, doch wurde der Aufsichtsrat in der Praxis nicht angenommen, Kalss/Burger/EcKert, aaO (Fn. 4), S. $265 \mathrm{ff}, 269$.

$12 \$ 111$ Abs. 4 Satz 1 AktG, dies zugleich mit dem neuen Prinzip der Satzungsstrenge; dazu Lieder, aaO (Fn. 4) S. $345 \mathrm{ff}, 353 \mathrm{ff}, 354$ : „Das nationalsozialistisch geprägte Schrifttum betonte, dass es neben dem Vorstand als „Führer des Unternehmens“ kein zweites Verwaltungsorgan geben dürfe.“ Aber nach Lieder, aaO, S. 356: klare Trennung der Kompetenzbereiche von Vorstand und Aufsichtsrat als wesentliches Verdienst der Aktienrechtsreform 1937. Auch Bayer/Engelke, in: Bayer/Habersack, Aktienrecht im Wandel, Bd. I, S. 619 Rdn. 71 ff; LutTer, in: Bayer/Habersack, Aktienrecht im Wandel, Bd. II, 2007, S. 389. 
der Praxis führte das zu einer Differenzierung zwischen verschiedenen Realtypen der Aktiengesellschaft: In den Familiengesellschaften und den Konzernen, die das Rückgrat der Wirtschaft bildeten, war es selbstverständlich so, dass die Familie bzw. die Muttergesellschaft den Aufsichtsrat mit ihren Vertrauenspersonen bestückte und über diese wesentlichen Einfluss auf den Vorstand ausüben konnte und ausübte. In den großen Aktiengesellschaften kam es, vorgeformt schon seit der Mitte des 19. Jahrhundert durch die Finanzierung und Vertretung der großen Banken in den Aufsichtsräten, zum später sogenannten rheinischen Kapitalismus. In der heutigen internationalen Typologie der Corporate Governance wird das als insider system bezeichnet gegenüber dem kapitalmarktorientierten outsider system. Die zunehmende Beteiligung von kleinen Aktionären änderte daran nichts. Aber auch in kleineren und mittleren Aktiengesellschaften war die Beratungsaufgabe des Aufsichtsrats von erheblicher Wichtigkeit. Gerade wenn es im Aufsichtsrat dort den der Familie Nahestehenden an besonderen Sachverständigen fehlte, war es an den Banken, finanziellen und anderen Rat zu erteilen. Auch heute noch haben in der Praxis der Familiengesellschaften die Aufsichtsräte ganz primär Beratungsfunktion, nicht oder weit weniger Überwachungsfunktion.

\section{Unternehmerische Mitbestimmung, Rückzug der Banken aus den Aufsichtsräten großer Gesellschaften und Aufsichtsratsreformen seit den 1990er Jabren}

Die großen Änderungen kamen in der zweiten Hälfte des letzten Jahrhunderts. Die erste war die Einführung der (quasi-)paritätischen Arbeitnehmermitbestimmung in den großen deutschen Unternehmen $1976 .{ }^{13} \mathrm{Ob}$ diese zu einem Bedeutungsverlust oder einem Bedeutungsgewinn des Aufsichtsrats geführt hat, lässt sich nicht allgemein beantworten. Richtig ist, dass in manchen Gesellschaften anfangs versucht wurde, die maßgeblichen Geschäfte aus dem Aufsichtsrat herauszuhalten, dies in Abstimmung mit dem von der Aktionärsseite bestellten Aufsichtsratsvorsitzenden. Auch die nicht selten vorkommende Durchbrechung des Unternehmensgeheimnisses aus dem Aufsichtsrat heraus, die oft zu Recht oder zu Unrecht nur der Arbeitnehmerseite angelastet wurde,${ }^{14}$ spielte dabei eine Rolle. Sie konnte allerdings auch durch die ausdrückliche gesetzliche Statuierung der Geheimhaltungspflicht 2002 nicht unterbunden werden. Das änderte sich tendenziell erst mit der Insiderrechtsgesetz-

13 MitbestG 1976; Oetker, Großkomm. z. AktG, Bd. 6, 5. Aufl., 2018. Zu \111 Abs. 4 Satz 1 AktG näher unten III. 1. b). Zur Entwicklung der (drittelparitätischen) Arbeitnehmermitbestimmung im Aufsichtsrat in Österreich $(1919,1947,1974)$ KALSs/Burger/ ECKERT, aaO (Fn. 4), S. $353 \mathrm{ff}$.

14 Auch für Österreich Kalss/Schauer, aaO (Fn. 2), S. 164. 
gebung. Auf der anderen Seite stellte sich später, spätestens mit dem Aufkommen der ersten feindlichen Übernahmen, die Einsicht ein, dass die Arbeitnehmer die besten Verbündeten von Vorstand und Aufsichtsrat in der Abwehr solcher Übernahmen und, wie prototypisch bei Volkswagen, auch sonst und allgemeiner waren. Die deutsche Umsetzung der europäischen Übernahmerichtlinie derart, dass der Aufsichtsrat das Sagen über die Zulässigkeit von Verteidigungsmaßnahmen bekam, ${ }^{15}$ trug dem Rechnung. Das dafür bemühte Argument, der Aufsichtsrat sei eine neutrale Überwachungsinstanz, löste im Ausland zu Recht Unverständnis aus.

Die zweite große Änderung war der Rückzug der Banken aus den Aufsichtsräten großer Gesellschaften im Zuge der Auflösung des rheinischen Kapitalismus. ${ }^{16}$ Eine Folge dieser Entwicklung war sicher, dass die mit durch die Banken und deren Sitz in den Aufsichtsräten der großen Gesellschaften begünstigte Vernetzung der Unternehmen und Wirtschaft zurückging, eine Entwicklung, die natürlich auch andere Ursachen wie das Erstarken des Kartellrechts hatte. Die Funktion des Aufsichtsrats als Dreh- und Informationszentrum über die einzelnen Unternehmen hinaus nahm damit ab. Schwer zu sagen ist, ob der Rückzug der Banken auch einen Verlust an Beratung durch den Aufsichtsrat zur Folge hatte oder ob diese sich dann, soweit die Banken betroffen waren, eben außerhalb fortsetzte. Was die Auswirkung auf die Überwachungsfunktion des Aufsichtsrats angeht, ist umstritten, inwieweit die Banken in den Aufsichtsräten zuvor eine effektive Überwachung ausgeübt hatten oder erst dann, wenn sich Unternehmenskrisen abzeichneten. ${ }^{17}$ Letztere blieb auch nach dem Rückzug der Banken aus den Aufsichtsräten schon angesichts des Eigeninteresses der Kreditbanken erhalten.

Mit der Zunahme der Aktionärsschaft wurde allmählich bewusst, dass jedenfalls der Aufsichtsrat seine Überwachungsfunktion vielfach nicht mehr richtig erfüllte und die deutsche Corporate Governance im internationalen Vergleich ganz erhebliche Schwächen hatte. Der deutsche Aufsichtsrat wurde nicht nur von Davies noch 2002 als „ein eher ineffektives Überwachungsorgan“ angesehen. ${ }^{18}$ Das rief 1998 den Gesetzgeber des KonTraG auf den Plan mit einer umfassenden Reform, darunter insbesondere auch der Verpflichtung zur Einrich-

15 \33 Abs. 1 Satz 2 WpÜG. Näher unten III. 3. b).

16 Ringe, Changing Law and Ownership Patterns in Germany: Corporate Governance and the Erosion of Deutschland AG, The American Journal of Comparative Law 63 (2015), 493; auch schon von HeIn, Die Rezeption US-amerikanischen Gesellschaftsrechts in Deutschland, 2008, S. $376 \mathrm{ff}$.

17 Zweifelnd Edwards/Fischer, Banks, finance, and investment in Germany, 1994, p. 196 et $s$.

18 Davies, ZGR 2001, 268, 292; Reformpostulate bei LutTer, ZHR 159 (1995), 287. Näher unten II. 2. b). 
tung eines Risikoüberwachungssystems. ${ }^{19}$ Seither ist es zur sogenannten Aktienrechtsreform in Permanenz gekommen, gesetzlich und ab 2002 auch durch den Deutschen Corporate Governance Kodex (DCGK). Letzterer bindet den Aufsichtsrat auch in die Entwicklung der strategischen Ausrichtung des Unternehmens ein..$^{20}$ International hatte sich demgegenüber schon lange die Auffassung durchgesetzt, dass der board die wichtigste Instanz für gute Corporate Governance ist. ${ }^{21}$ Auch die höchstrichterliche Rechtsprechung tat mit dem ARAG/Garmenbeck-Urteil 1997 das Ihrige dazu und verpflichtete den Aufsichtsrat, durchsetzbare Schadensersatzansprüche gegen den Vorstand grundsätzlich zu verfolgen. Davon abzusehen blieb dem Aufsichtsrat seither nur noch in engen Grenzen möglich, und dann unter dem Damoklesschwert seiner eigenen Haftung. ${ }^{22}$ Diese Rechtsprechung und der mit der Finanzmarktkrise verbundene Vertrauensverlust der Öffentlichkeit in Finanz, Politik und Institutionen führten seither zu einer deutlichen Zunahme der Haftungsprozesse. Seit 2002 hat die Satzung oder der Aufsichtsrat auch zu bestimmen, dass bestimmte Arten von Geschäften nur mit seiner Zustimmung vorgenommen werden dürfen, ${ }^{23}$ eine weitere Aufwertung des Aufsichtsrats. Zuletzt scheint es interessierten Kreisen, darunter den Gewerkschaften, politisch gelungen zu sein, eine Verlagerung der Zuständigkeit für die Vorstandsvergütung auf die Hauptversammlung durch einen verbindlichen say on pay zu verhindern, und die Umsetzung der Vorgaben der Aktionärsrechte-Richtlinie zu den related party transactions führt voraussichtlich zu einer weiteren Aufwertung des Aufsichtsrats.

Die bisherigen Beobachtungen haben ergeben, dass der Aufsichtsrat in den letzten Jahrzehnten ganz erheblich an Bedeutung gewonnen hat, auch wenn in der Öffentlichkeit manchmal der falsche Eindruck eines breitflächigen Versagens erweckt wird. Das lässt sich jedenfalls für Deutschland feststellen, gilt aber auch für Österreich, ${ }^{24}$ bei aller berechtigten Kritik am Aufsichtsrat. ${ }^{25}$ Die Feststellung dieses Bedeutungszuwachses ist aber nur ein Zwischenergebnis. Denn er gilt nur für das zweistufige System von Vorstand und Aufsichtsrat, das international in der Minderheit steht. Das könnte anders sein, wenn sich der Aufsichtsrat, also das zweistufige System im Verhältnis zum einstufigen

19 \91 Abs. 2 AktG. Wie weit das rechtlich geht bzw. gehen müsste, ist zwischen der Rechtswissenschaft und der Betriebswirtschaftslehre streitig, Nachweise bei KocH, aaO (Fn. 1), $\$ 91$ AktG Rdn. 8 ff. Dazu BerenbroK, Risikomanagement im Aktienrecht, 2016.

20 DCGK 2017 Ziff. 3.2. Im DCGKE 2019 als Grundsatz 4 weiter enthalten.

21 Hopt, ZHR 175 (2011), 444, 465 ff. Auch Bachmann, FS Hopt, Bd. 1, 2010, S. 337.

22 Zur ARAG/Garmenbeck-Rechtsprechung unten III. 1. a).

$23 \rrbracket 111$ Abs. 4 Satz 2 AktG, eingeführt durch Gesetz vom 19.7.2002, BGBl. I, S. 2681.

24 Umfassend Kalss/Kunz, Handbuch für den Aufsichtsrat, 2. Aufl., 2016.

25 P. Doralt, FS Hopt, Bd. 2, 2010, S. 3059. 
des board bzw. Verwaltungsrats, nicht nur behauptet, sondern wenn sich die Systeme tatsächlich aufeinander zu bewegen, also eine gewisse Konvergenz festzustellen ist.

\section{Konvergenz des ein- und des zweistufigen Systems bei verbleibenden Pfadabhängigkeiten}

Voranzuschicken ist, dass das einstufige System (Verwaltungsrat, board) herkömmlich überwiegt, z.B. in Belgien, England, Frankreich, Irland, Spanien und der Schweiz, während Deutschland, Österreich, Portugal, Polen, Slowakei, Tschechien und Ungarn herkömmlich nur das dualistische System (Vorstand und Aufsichtsrat) kennen, einige Länder wie die Niederlande, Slowenien und Dänemark haben Mischformen. ${ }^{26}$ Verbreitet ist inzwischen das Wahlrecht zwischen beiden oder mehr Systemen. ${ }^{27}$

Das zweistufige System könne sich auf Dauer nicht halten, so die Behauptung von Henry Hansmann und Reinier Kraakman in einem viel beachteten Aufsatz von 2001 über „The End of History for Corporate Law“. ${ }^{28}$ Begründet haben die beiden Autoren aus Yale und aus Harvard das mit den der Globalisierung und den internationalen Marktkräften, kraft derer sich das effizientere System durchsetzt. Dabei gingen sie - man erlaube mir die Spitze - wie viele unserer amerikanischen Freunde ohne weiteres davon aus, dass das eigene amerikanische, hier also das einstufige System das effizientere ist. Tatsächlich hat sich aber in den letzten 20 Jahren nichts dafür ergeben, dass der Aufsichtsrat zu seinem geschichtlichen Ende gekommen wäre. Vielmehr sind die Länder, die traditionell entweder dem ein- oder dem zweistufigen System anhängen, trotz zahlreicher Reformen im Aktien- und Kapitalmarktrecht jeweils bei dem ihrigen geblieben. Warum das so ist, haben Paul Davies und ich in einem Aufsatz im American Journal of Comparative Law $2013^{29}$ näher begründet,

26 Hopt/Leyens, ECFR 2004, 135; Hopt, ZHR 175 (2011), 444, 466ff; Kalss/Schauer, $\mathrm{aaO}$ (Fn. 2), S. 91.

27 Hopt, ZHR 175 (2011), 444, $468 \mathrm{f}$.

28 Hansmann/Kraakman, The End of History for Corporate Law, 89 Geo. L. J. 439 (2001); Hansmann, How Close is the End of History? 31 Journal of Corporate Law 745 (2006); modifiziert, aber in der Sache aufrechterhalten später Hansmann/KraAkman, Reflections on the End of History for Corporate Law, in: Rasheed/Yoshikawa, The Convergence of Corporate Governance, 2012, p. 32. Soeben allgemeiner GoshEN/HaNNES, The Death of Corporate Law, New York University Law Review 94 (2019) 263: shift of regulatory power from (Delaware) courts to markets.

29 Davies/Hopt, Corporate Boards in Europe - Accountability and Convergence, The American Journal of Comparative Law 61 (2013), 301-375; dies auf der Grundlage eines internationalen Forschungsprojekts: Davies/Hopt/NowaK/van Solinge, eds., Corporate Boards in Law and Practice, A Comparative Analysis in Europe, Oxford 2013, mit 
was hier nicht wiederholt werden soll. Die bloß ökonomische Prognose reicht eben nicht aus, die nationalen und internationalen Marktkräfte richten sich nicht allein an Effizienz aus. Vielmehr vollziehen sich Gesetzesreformen und Änderungen in Rechtssystemen komplexer im politischen Zusammenspiel derer, die dabei gewinnen oder verlieren, ihrer Lobby, der Öffentlichkeit und der Gesetzgeber im jeweiligen Land und in Brüssel. ${ }^{30}$

Stattdessen lässt sich, wie im Folgenden auszuführen, international eine Konvergenz zwischen dem ein- und dem zweistufigen System feststellen, auch wenn eine Reihe von Divergenzen oder, wie es im Jargon heißt, Pfadabhängigkeiten verbleiben. ${ }^{31}$ Das ist wohl gemerkt eine funktionale Aussage, rein nach Recht und Begriff wird es beim Verwaltungsrat und beim Aufsichtsrat bleiben. Konvergenz und Pfadabhängigkeiten zeigen sich in drei Bereichen: der Entscheidungsfindung, der Überwachung und der Zusammensetzung der Mitglieder. Dabei fällt der Blick zunächst auf die Konvergenzen im einstufigen System mit dem zweistufigen und dann auf die Pfadabhängigkeiten im zweistufigen im Vergleich zum einstufigen.

\section{Konvergenzen}

\section{a) Entscheidungsfindung}

Die international, aber auch in Deutschland immer noch zu findende Meinung, der Verwaltungsrat im einstufigen System entspreche einem allzuständigen Vorstand und der Aufsichtsrat sei im Wesentlichen ein bloßes Überwachungsorgan, hält dem tatsächlichen Befund nicht stand. Die Entscheidungsfindung

Buchbesprechungen von WymEersch, NZG 2016, 938 und Journal of Corporate Law Studies 17 (2017), 253, sowie HiRT, AG 2017, 715.

30 Zur Bedeutung der politischen Kräfte für das Aktienrecht schon früh RoE, Strong Managers, Weak Owners, The Political Roots of American Corporate Finance, 1994, und in vielen weiteren Beiträgen seither. Internationaler Milhaupt/Pistor, Law \& Capitalism, What Corporate Crises Reveal about Legal Systems and Economic Development around the World, 2008.

31 Zur Konvergenz der beiden Systeme statt vieler BöcKLI, aaO (Fn. 9), S. 255; auch DERs., aaO (Fn. 1), $\mathbb{S} 13 \mathrm{Rdn} .535 \mathrm{ff}, 968 \mathrm{ff}$; von HeIn, aaO (Fn. 16), S. $42 \mathrm{f}$ mit umfangreichen Nachweisen auch schon aus der Weimer Zeit, S. 238, 259ff, allgemeiner zur Konvergenzthese, S. 33 ff, 796ff. Auch schon Conard, ZGR 1987, 180; Нopt, ZGR 2000, 779, 788 f; DERS., Aktienrecht unter amerikanischem Einfluss, FS Canaris, Bd. 2, 2007, S. 105, 111; DERS., Corporate Governance und Krise: Verwaltungsrat und/oder Vorstand und Aufsichtsrat in Europa, in: Binder/Psaroudakis, Europäisches Privat- und Wirtschaftsrecht in der Krise, 2018, S. 269; Leyens, FS Hopt, Bd. 2, 2010, S. 3135; Норт/Roth, aаO (Fn. 1), $₫ 111$ AktG Rdn. 89 ff. Für die Schweiz allgemeiner VogT, Konvergenz von Gesellschaftsrechten, 2012. Für Österreich Kalss, aaO (Fn. 1), Vor $\$ 86$ AktG Rdn. 25. 
im Unternehmen ist komplexer. In größeren Aktiengesellschaften, zumal global tätigen und konzernierten, ist der Verwaltungsrat gar nicht mehr imstande, das ganze Unternehmen selbst zu leiten. Vielmehr findet rechtstatsächlich in breitem Umfang eine Delegation auf das Management statt, der Verwaltungsrat gibt nur die Grundlinien vor und überwacht deren Einhaltung. Dass der Verwaltungsrat zwar die Befugnis zur Geschäftsführung hat, diese aber tatsächlich nicht oder nur begrenzt ausübt, ist aus den USA und dem Vereinigten Königreich bekannt (monitoring board). ${ }^{32}$ Aus der Schweiz wird berichtet, dass es seit längerer Zeit weit verbreitet ist, die Geschäftsführung weitgehend einem eigenen „Organ“, der Geschäftsleitung, zu übertragen. Die Geschäftsführung als weithin eigenständig funktionierendes Gremium gibt es in der Schweiz nicht nur in den größten Aktiengesellschaften, sondern hat sich auch

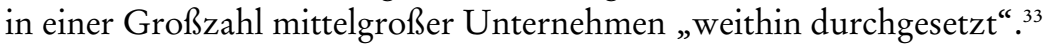

\section{b) Überwachung}

Dass es um einstufigen System kein eigenes Überwachungsorgan wie den Aufsichtsrat gibt, zwar formal richtig, insoweit ein solches nicht gesetzlich vorgesehen ist. Bis in die zweite Hälfte der Achtziger Jahre hatte der board nach britischem Gesellschaftsrecht nicht einmal als Ganzes, für die Überwachung der Gesellschaft zu sorgen, das war Sache der Aktionäre. ${ }^{34}$ Das hat sich mit den Corporate Governance-Reformen geändert, obschon es im Vereinigten Königreich und in der Schweiz bei einem einzigen Organ geblieben ist. Aber die Trennung zwischen Leitung und Überwachung, so der Doppelbegriff der Corporate Governance nach der Cadbury Commission 1992,35 findet heutzutage gerade in den anglo-amerikanischen Ländern im Verwaltungsrat selbst statt. Dort haben die independent directors im board die Aufgabe, für die Überwachung der anderen Direktoren zu sorgen und dabei vor allem auf Interessenkonflikte zu achten. ${ }^{36}$ Wenn danach in bestimmten kritischen Fällen

32 Für die USA P. Doralt/W.Doralt, in: Semler/v. Schenck, Arbeitshandbuch für Aufsichtsratsmitglieder, 4. Aufl., 2013, $\mathbb{1 4}$ Rdn. 86. Für den britischen board Davies/ Worthington, aaO (Fn. 10), 14-3, 14-10: delegation to senior management of enormous importance in large companies, 14-65: practice increasingly common.

33 Böckli, aaO (Fn. 1), $\mathbb{1} 13$ Rdn. 535 ff, 539; Nobel, aaO (Fn. 1), $\$ 9$ N. 111: alle größeren Gesellschaften kennen heute eine „Direktion“. Aber Art. 716a OR zu unübertragbaren und unentziehbaren Aufgaben, dazu BüHLER, in: Handschin, Die Aktiengesellschaft (Zürcher Kommentar), 3. Aufl., 2018, Art. 716a N 43: Zweiteilung in der Praxis der Regelfall.

34 DAvies, ZGR 2001, 268, 292.

35 Cadbury, Report of the Committee on the Financial Aspects of Corporate Governance, December 1992.

36 Dazu Hopt, Der neue UK Corporate Governance Code 2018 - mit Denkanstößen für die Reform des Deutschen Corporte Governance Kodex -, FS Seibert, 2019, im Druck. 
nur die independent directors entscheiden können und die entsprechenden Ausschüsse mehrheitlich oder sogar ausschließlich mit solchen Direktoren besetzt werden dürfen, entspricht das insoweit durchaus der Trennung von Vorstand und Aufsichtsrat im zweistufigen System, ${ }^{37}$ ja mehr noch, diese Trennung wird durch ein echtes Unabhängigkeitserfordernis konsequenter durchgeführt. Hinzu kommt prototypisch im Vereinigten Königreich die Trennung des chief executive officer (CEO) und des chair(man) of the board, was jedenfalls nach dortiger Vorstellung allein guter Corporate Governance entspricht.

\section{c) Zusammensetzung}

Eine Konvergenz im board bzw. Verwaltungsrat hin zu Differenzierung und Trennung jedenfalls innerhalb der Organe gibt es nicht nur, wie ausgeführt, zwischen CEO und chairman of the board und durch die Bildung und besondere Aufgabenstellung der independent directors. Auch allgemeiner wird dort wie in den zweistufigen Systemen eine Geschlechterquote durchgesetzt und darüber hinaus breiter eine Diskussion um die Diversität des Organs geführt, was die corporate governance verbessern, aber auch neue Trennungen hervorrufen kann.

\section{Divergenzen (Pfadabhängigkeiten)}

\section{a) Entscheidungsfindung}

Im zweistufigen System leitet der Vorstand die Gesellschaft unter eigener Verantwortung, der Aufsichtsrat hat die Geschäftsführung zu überwachen. ${ }^{38}$ Rechtlich hat der Aufsichtsrat aber eine ganze Reihe ihm vom Gesetz ausdrücklich zugewiesener weiterer Einzelbefugnisse, und in einer ganzen Reihe von Fällen kann der Vorstand nur zusammen mit dem Aufsichtsrat tätig werden, Beispiele sind die Erklärungen und Stellungnahmen nach $\ 161$ Abs. 1 AktG, $\mathbb{2} 289 \mathrm{a}$ HGB und $\ 27$ Abs. 1, 3 WpÜG. ${ }^{39}$ In einer ganzen Reihe ande-

37 Im Ergebnis auch v. Werder, DB 2017, 977, 978, aber genauer genommen sind es nicht alle non-executive directors (NEDs), die das Äquivalent zum Aufsichtsrat bilden, sondern die independent (non-executive) directors. Im ökonomischen Schrifttum wird die Parallele zwischen dem Prüfungsausschuss im Verwaltungsrat und dem Aufsichtsrat gezogen; Adams/Ferreira, A Theory of Friendly Boards, 62 Journal of Finance 217, 235 (2007): “(T)he role of the audit committee in the sole board systems of the United States and the United Kingdom may be similar to that of supervisory boards".

38 Für den Vorstand $\$ 76 \mathrm{AktG}$, dazu Koch, aaO (Fn. 1), $\$ 76 \mathrm{AktG}$ Rdn. 6 ff. Für den Aufsichtsrat $\mathbb{1 1 1}$ Abs. 1 AktG, Косн, aaO, $\mathbb{1 1 1}$ AktG Rdn. 2 ff: „Der Aufsichtsrat als Überwachungsorgan“.

39 Dazu Hirt/Hopt/Mattheus, AG 2016, 725, 732. 
rer Fälle hat der Aufsichtsrat eigene unternehmerische Entscheidungen zu treffen. ${ }^{40}$ Hinzu kommt die 2002 eingeführte zwingende Vorschrift des $\ 111$ Abs. 4 Satz 2 AktG, dass die Satzung oder der Aufsichtsrat zu bestimmen hat, dass bestimmte Arten von Geschäften nur mit seiner Zustimmung vorgenommen werden dürfen. Im Übrigen werden für den Aufsichtsrat ganz ähnliche Anforderungen wie für den board gestellt oder gefordert, etwa an die Ausschussbildung, den Zeitaufwand, die Befassung mit Strategie, Risikomanagement und interner Kontrolle und Evaluation. ${ }^{41}$ Auch die neuerliche Diskussion um die Unternehmensziele, also mehr stakeholder-Orientierung, wird unter beiden Systemen geführt.

Als Pfadabhängigkeit verbleibt, dass der deutsche Gesetzgeber ausdrücklich bestimmt hat, dass Maßnahmen der Geschäftsführung dem Aufsichtsrat nicht übertragen werden können ( $\mathbb{S} 111$ Abs. 4 Satz $1 \mathrm{AktG})$. Ausdrückliche Grenzen der Delegation gibt es auch umgekehrt vom Verwaltungsrat auf das Management. Ein Kernbestand von Aufgaben muss der Verwaltungsrat selbst wahrnehmen, namentlich die Oberleitung des ganzen Unternehmens, die Organisations- und Finanzverantwortung und die Wahl der Geschäftsleitung. ${ }^{42}$ Im zweistufigen System verbleibt Vorstand ,in the driving seat“, auch wenn der Aufsichtsrat überwacht und mitsteuert, man kann insofern mit Hommelhoff von „Kooperation in kritischer Distanz“ sprechen. Das wird besonders deutlich im Konzern, in dem der Vorstand der Muttergesellschaft den Ton angibt, obschon es rechtlich keinen Konzernvorstand gibt, aber erst recht eben auch keinen Konzernaufsichtsrat. Diese unterschiedliche Gewichtung spiegelt sich auch in den riesigen Unterschieden in der Vergütung von Vorständen und Aufsichtsräten. ${ }^{43}$

\section{b) Überwachung}

Paul Davies hat vor bald zwanzig Jahren in einem Geburtstagssymposion für mich die Frage gestellt: „Struktur der Unternehmensführung in Großbritannien und Deutschland: Konvergenz oder fortbestehende Divergenz? "“44 Nach sorg-

40 Ausführlich Lieder, ZGR 2018, 523.

41 Davies/Hopt, The American Journal of Comparative Law 61 (2013), 301 at 314 et seq.

42 Böckli, aaO (Fn. 1), $\int 13$ Rdn. 542.

43 Allen \& Overy, Die Vorstands- und Aufsichtsratsgehälter der DAX-30-Unternehmen, 2018; PWC zusammen mit BöCKING U.A., Vergütungsstudie 2018, Vorstands- und Aufsichtsratsvergütung im Dax, MDax, SDax und TecDax 2014-2017, dort S. 27 mit einem Plädoyer für die Stärkung der Aufsichtsräte durch adäquate Vergütungssysteme; BöCKING U. A., Der Konzern 2019, 15. Zum ARUG II-RefE BAYER, DB 2018, 3024.

44 Davies, ZGR 2001, 268. Zum Vergleich des deutschen und englischen Systems auch LeYENS, Information des Aufsichtsrats, Ökonomisch-funktionale Analyse und Rechtsvergleich zum englischen Board, 2006. 
fältiger Vergleichung meinte er feststellen zu können, dass der deutsche Aufsichtsrat ein eher ineffektives Überwachungsorgan ist, während der britische board die Überwachungsaufgabe nicht nur hat, sondern in der Praxis besser umsetzt, was vor allem an dem ineffizienten Informationsfluss vom Vorstand zum Aufsichtsrat liegt. ${ }^{45}$ Wenn es aber, wie vorher zur Entscheidungsfindung ausgeführt, auch im einstufigen System häufig so ist, dass der board die Geschäftsführung weitgehend an das Management delegiert, dann stellt sich oben erörterte Informationsproblem des zweistufigen auch im einstufigen System, allerdings dann gemindert, wenn wie in den USA und Frankreich der CEO bzw. PDG zugleich dem Verwaltungsrat vorsitzt. ${ }^{46}$ Umgekehrt hat sich beim deutschen Aufsichtsrat und der deutschen Corporate Governance seit dem KonTraG $1998^{47}$ mit den nicht weniger als 54 aktienrechtlichen Reformgesetzen seither und dem Deutschen Corporate Governance Kodex zumal unter dem Eindruck der Finanzkrise und unter einer strengeren höchstrichterlichen Rechtsprechung nach dem ARAG/Garmenbeck-Urteil von 1997 vieles gebessert.

Als Pfadabhängigkeit bleibt aber dennoch, dass beim Aufsichtsrat das gesetzgeberische Modell die Informationsverantwortlichkeit beim Vorstand sieht. Die wohl noch immer überwiegende Meinung steht der sogenannten vorstandsunabhängigen Information ablehnend gegenüber, auch wenn sich das unter dem Eindruck internationaler Befunde, wie weiter unten auszuführen, ${ }^{48}$ allmählich ändert.

\section{c) Zusammensetzung}

Die zum einstufigen System festgestellte Entwicklung hin zu Differenzierung und Trennung findet sich auch beim Aufsichtsrat, hier mit einer zwingenden Inkompatibilität, die durch die allerdings umstrittene Karenzzeitregelung von zwei Jahren für den Wechsel vom Vorstand in den Aufsichtsrat 2009 (\$100 Abs. 2 Satz 1 Nr. 4 AktG) verschärft worden ist. Wie im einstufigen System gibt es mittlerweile auch mehr oder weniger weit gehende Anforderungen an unabhängige Aufsichtsratsmitglieder, wenn auch in Deutschland bislang nur auf der Ebene des Deutschen Corporate Governance Kodex. Demgegenüber ist eine 30 \% ige Zielgrößen-Geschlechterquote auch in Deutschland 2015 Gesetz geworden ${ }^{49}$ Aber im politischen Raum werden Stimmen laut, diese auch auf den Vorstand auszudehnen und die Anforderungsschwelle zu erhöhen.

46 Dazu unten II. 3. c).

47 Kontroll- und Transparenzgesetz (KonTraG) vom 27.4.1998, BGBl. I, S. 786. Näher Hopt, AG Sonderheft August 1997, 42; DERs, Freundesgabe für Kübler, 1997, S. 435.

$49 \int \mathbb{S} 111$ Abs. 5, 76 Abs. 4 AktG. 
Pfadabhängigkeiten verbleiben. Das trifft schon beim board-System selbst zu. So findet sich die Trennung von CEO und chairman of the board zwar im Vereinigten Königreich, aber in den USA ist der CEO Mitglied im board ${ }^{50}$ und in Frankreich ist der président directeur général (PDG) gleichzeitig dirigeant de la SA und président du conseil d'administration, ${ }^{51}$ was angesichts von Satzungsfreiheit auch in der Schweizer Praxis vorkommt. ${ }^{52}$ Beide Systeme, das einstufige und das zweistufige, haben ökonomisch Vor- und Nachteile. ${ }^{53}$ Fundamental ist dagegen die Sonderstellung des deutschen - nicht auch des österreichischen ${ }^{54}$ - Aufsichtsrats mit seiner quasi-paritätischen unternehmerischen Mitbestimmung. ${ }^{55}$ Dieser Sonderweg geht auf die besondere Situation Deutschlands nach dem ersten und dem zweiten Weltkrieg zurück und ist heute politisch tabuisiert. Welche ökonomischen Konsequenzen dieser Sonderweg hat und ob die Nachteile durch die Vorteile kompensiert werden, ist trotz einer ganzen Anzahl empirischer Untersuchungen bislang nicht geklärt. Wissenschaft und Praxis fordern schon lange mehr Flexibilität und Optionalität. Aber angesichts fester Besitzstände bis hin zur weitgehenden Abführung der Aufsichtsratsvergütungen an die gewerkschaftsnahe Hans-Böckler-Stiftung ist das System in Deutschland politisch festgezurrt und durch die jüngste Rechtsprechung des Europäischen Gerichtshofs bestätigt worden. International gibt es zwar immer wieder vorsichtige Ansätze zur Einführung von Mitbestimmung, zuletzt in den $\mathrm{USA}^{56}$ und im Vereinigten Königreich ${ }^{57}$, aber sie sind schon in ihrer gegenüber Deutschland höchst bescheidenen Fassung dort politisch chancenlos.

50 P. Doralt/W. Doralt, aaO (Fn. 32), $\ 14$ Rdn. 87.

51 Näher Cozian/Viandier/Deboissy, Droit des sociétés, 23ième éd., 2010, p. 276 et s., 320 et s., zu directoire et conseil de surveillance, p. 349 et s., im CAC 40 ungefähr $20 \%$ der Gesellschaften, p. 350; Pietrancosta/Dubois/Garçon, in: Davies/Hopt/Nowak/van Solinge, Corporate Boards in Law and Practice, A Comparative Analysis in Europe, 2013, p. 178, 184 et s., 196 et s., 203, 244 et s.

52 Meier-Hayoz/Forstmoser/Sethe, aaO (Fn. 1), $\mathbb{} 16$ N. 586, aber nicht mit derselben Machtfülle in der Hand einer Einzelperson.

53 Bainbridge, The Board of Directors, in: Gordon/Ringe, The Oxford Handbook of Corporate Law and Governance, Oxford 2018, ch. 12, p. 275, 321 et seq.

54 Zur dortigen Drittelbeteiligung Kalss, aaO (Fn. 1), $\$ 86$ AktG Rdn. 28 ff; Gahleitner, in: Kalss/Kunz, Handbuch für den Aufsichtsrat, 2. Aufl., 2016, $\$ 10$.

55 Schon oben I. 3. mit Fn. 13; Böckli, aaO (Fn. 1), $\$ 13$ Rdn. 989 spricht, seine Überlegungen zur Konvergenz der Systeme abschließend, von einer „offenbar unüberwindliche(n) Divergenz“; auch НOPT, ZGR 2000, 779, $798 \mathrm{ff}$ und schon DERs., Arbeitnehmervertretung im Aufsichtsrat - Auswirkungen der Mitbestimmung auf corporate governance und wirtschaftliche Integration in Europa, FS Everling, 1995, S. 475.

56 Gesetzesvorschlag der Abgeordneten im Repräsentantenhaus Elizabeth Warren, Accountable Capitalism Act, Senate Bill 3348, abrufbar unter: https://www.congress.gov/ bill/115th-congress/senate-bill/3348/text (letzter Zugriff: 4.6.2019). 
Funktional gesehen überwiegt nach alledem trotz mancher pfadabhängiger Unterschiede insgesamt die Konvergenz. ${ }^{58}$ Dabei sollte heute klar sein, dass keines der beiden Systeme besser ist, auch ökonomisch-empirisch gibt es dazu keine Nachweise ${ }^{59}$. Sie sind nur unterschiedlich geeignet für die konkreten Unternehmen. Die logische Konsequenz ist ein Wahlrecht zwischen den Systemen, wie es in vielen Mitgliedstaaten der Europäischen Union im Anschluss an die Regelung für die Europäische Gesellschaft (SE) besteht. ${ }^{60}$ Der deutsche Gesetzgeber hat sich dem trotz vielfacher Aufforderung, auch des Deutschen Juristentags, ${ }^{61}$ bisher verschlossen, vordergründig wegen des Kodifikationsaufwands, tatsächlich aber doch wohl maßgeblich im Hinblick auf die Mitbestimmung. ${ }^{62}$ Meines Erachtens wäre ein solches Wahlrecht ein willkommener Schritt zu mehr Flexibilität, Satzungsfreiheit und Wettbewerb der Systeme.

Die Betrachtungen zur Konvergenz haben gezeigt, dass der Aufsichtsrat, genauer: das zweistufige System, auch international mitnichten als bloßes Minderheitsmodell zurückstehen muss. Ganz im Gegenteil werden hier die Vorteile der klaren Trennung von Leitung (direction) und Überwachung (control), den beiden Eckpfeilern jeder Corporate Governance, insoweit durchaus exemplarisch und ausstrahlend, in der Unternehmenspraxis vorgelebt. Die Konvergenz festzustellen, bedeutet allerdings nicht, dass die Grenzziehung zwischen den beiden Modellen immer einfach und unumstritten wäre, im Gegenteil. Im Folgenden ist das für das deutsche Recht an drei Beispielen aufzuzeigen, diese sind die unternehmerischen Entscheidungen des Aufsichtsrats, das Verhältnis von Aufsichtsrat und Information und der Aufsichtsrat und die Interessenkonflikte.

58 Davies/Hopt, aaO (Fn. 29), The American Journal of Comparative Law 61 (2013), 301 at 312 et seq.; BöckLI, aaO (Fn. 1), $\$ 13$ Rdn. 968 ff: Prozess mit bemerkenswerter Folgerichtigkeit.

59 Ausführlich Jungmann, ECFR 2006, 426; BAINBRIDGE, aaO (Fn. 53), ch. 12, p. 275, 289: "the choice between unitary and dual boards appears to be mainly a function of historical accident, inertia, or political bargaining rather than one of economic advantage”. Für Kreditinstitute ist die Trennung möglicherweise doch vorteilhaft, vgl. EuG, 24.4.2018, T-133/16 - T-136/16 (Crédit Agricole), zu Art. 88(1)(e) der Richtlinie 2013/36/EU, 26.6.2013 (Zulassung und Beaufsichtigung von Kreditinstituten): verantwortlicher Geschäftsleiter eines Kreditinstituts, keine Kumulation von Aufsichts- und Managementfunktionen, aber das ist auch in einem Verwaltungsrat durch entsprechende Organisation der Zuständigkeiten möglich, ebenda Rdn. $81 \mathrm{f}$.

60 SE-Verordnung vom 8.10.2011, ABl.EU L 294/1, Artt. 38, $39 \mathrm{ff}$ (zweistufig), $43 \mathrm{ff}$ (einstufig), $46 \mathrm{ff}$ (gemeinsame Vorschriften).

61 69. DJT 2012, Beschluss 19, abgedruckt in ZIP 2017, 1987, 1988; früher Hopt/LeYens, ECFR 2004, 135, 163 ff; Норт, ZHR 175 (2011), 444, 468f; weitere Nachweise bei Hopt/ Roth, aaO (Fn. 1), $\mathbb{9} 95$ AktG Rdn. 44. Für Österreich Kalss/Schauer, aaO (Fn. 2), S. $94 \mathrm{ff}$.

62 Ebenso für Österreich Kalss, aaO (Fn. 1), Vor $\$ 86$ AktG Rdn. 25. 
III. Die unternehmerische Mitentscheidung: Aufgaben des Aufsichtsrats zusammen mit dem Vorstand und allein

\section{Prospektive Überwachung und unternehmerische Entscheidungen des Aufsichtsrats}

\section{a) Prospektive Überwachung}

Der Aufsichtsrat wurde und wird als Überwachungsorgan verstanden. Überwachung wird herkömmlich, wie schon vom Wortlaut her nahegelegt, als Überwachung ex post verstanden. Daneben steht aber nach allgemeiner Ansicht die Beratung des Vorstands durch den Aufsichtsrat. Der Aufsichtsrat war schon immer und ist weiterhin auch ein Beratungsorgan. Beratung ist schon ihrem Wesen nach prognostisch. Aber auch Überwachung erst nachträglich kommt häufig zu spät. Die heute herrschende Meinung sieht die Überwachungsaufgabe des Aufsichtsrats deshalb zu Recht als weitergehend an und erkennt auch eine präventive, zukunftsbezogene Überwachung an. In dem oben erwähnten ARAG/Garmenbeck-Urteil hat der Bundesgerichtshof ausdrücklich die Fälle erwähnt, in denen das Gesetz auch dem Aufsichtsrat unternehmerische Aufgaben übertragen hat, nämlich „überall dort, wo er die unternehmerische Tätigkeit des Vorstands im Sinne einer präventiven Kontrolle begleitend mitgestaltet" ${ }^{63}$ Der Aufsichtsrat muss, wie heute allseits anerkannt, durch Kontrolle und Beratung von vornherein dafür sorgen, dass die Gesellschaft sachgerecht geleitet wird. Diese zukunftsgerichtete Kontrolle hat mit der zunehmenden Professionalisierung des Aufsichtsrats seit Anfang der 2000er Jahre ganz wesentlich an Bedeutung gewonnen. ${ }^{64}$

\section{b) Unternehmerische Aufgaben}

Die durchaus zahlreichen Fälle, in denen der Aufsichtsrat von Gesetzes wegen unternehmerische Aufgaben hat, nicht nur zusammen mit dem Vorstand, sondern allein, hat jüngst Lieder aufgeführt, darauf ist zu verweisen. ${ }^{65}$ Der Streit in

63 BGHZ 135, 244, 254 f. Auch schon 1991 BGHZ 114, 127, 129/130: „Die Kontrolle bezieht sich nicht nur auf abgeschlossene Sachverhalte, sondern erstreckt sich auch auf grundsätzliche Fragen der künftigen Geschäftspolitik; ... die Beratung ist deshalb das vorrangige Mittel der in die Zukunft gerichteten Kontrolle des Vorstands.“

64 Zutr. Lieder, ZGR 2018, 523, 524. Vgl. auch die Bestandsaufnahme der verschärften Überwachungsaufgaben des Aufsichtsrats bei BINZ/SORG, BB 2019, 387.

65 Lieder, ZGR 2018, 523, 533 ff. Auch schon Roth, ZGR 2012, 343, 348 f: „mit-unternehmerisches Organ“. Ebenso für den österreichischen Aufsichtsrat Kalss, aaO (Fn. 1), \95 AktG Rdn. 9: unternehmerische Mitverantwortlichkeit, obschon nicht Geschäftsführungsorgan, Rdn. $15 \mathrm{ff}$ strategische Begleitung. 
der Rechtswissenschaft findet heute im Wesentlichen nicht mehr dazu statt, sondern zu der Frage, ob der Aufsichtsrat in all diesen Fällen oder nur in einzelnen bei der Erfüllung seiner Aufgabe einen Ermessensspielraum im Sinne der business judgment rule hat. Das ist vor allem bei der Schaffung eines Mindestkatalogs zustimmungspflichtiger Geschäfte nach $\$ 111$ Abs. 4 Satz 2 AktG ohne weiteres zu bejahen. Das Gesetz verpflichtet den Aufsichtsrat zwar zum Tätigwerden, also zum Ob, aber nicht zur konkreten Ausgestaltung, dem Wie. Diese ist eine unternehmerische Entscheidung, wobei wie bei jedem Ermessen dieses in besonderen Fällen auf null schrumpfen kann. ${ }^{66}$ Das gilt erst recht hinsichtlich der Erteilung oder Nichterteilung einer solchen Zustimmung im konkreten Fall. Streitiger ist das bei der Frage der Abberufung eines Vorstandsmitglieds wegen eines wichtigen Grundes. Die h.L. geht zu Recht davon aus, dass der wichtige Grund objektiv vorliegen muss, dem Aufsichtsrat also insoweit zwar kein Beurteilungsspielraum zusteht, ${ }^{67}$ aber dass er dann ein Abberufungsermessen hat. ${ }^{68}$ Die Minderheitsmeinung argumentiert umgekehrt, also Beurteilungsspielraum hinsichtlich des wichtigen Grunds, dann aber Pflicht zu Abberufung. ${ }^{69}$ Im Endergebnis wird das vielfach keinen Unterschied machen. Insgesamt wird man sagen können: Die Abberufung eines Vorstands ist ein Unterfall der (vergangenheitsbezogenen und prospektiven) Überwachung. ${ }^{70}$ Noch streitiger ist die Frage bei der Vorstandsvergütung. Teils wird vertreten, dass der Aufsichtsrat hier ein unternehmerisches Ermessen hat, teils wird ihm nur ein Beurteilungsspielraum zugestanden, teils wird ein Unterschied gemacht, ob es sich um die originäre Vergütungsfestsetzung des Aufsichtsrats handelt oder um die Herabsetzung der Bezüge bei Verschlechterung der Lage der Gesellschaft. ${ }^{71}$ In einer nicht unproblematischen Grundsatzentscheidung hat der II. Zivilsenat des Bundesgerichtshofs eine sehr enge Meinung dazu vertreten und dem Aufsichtsrat nur die Befugnis zur „Korrektur auf einen (gerade noch) der Billigkeit entsprechenden Betrag " zugestanden. ${ }^{72}$ Demgegenüber hat sich zuletzt Lieder für einen unternehmerischen Ermessensspielraum ausgesprochen. Dieser Meinungsstreit hat hier auch praktisch Konsequenzen, zwar kaum für die originäre Vergütungsentscheidung, sehr wohl aber bei der

66 Zutr. ebenda Lieder, ZGR 2018, 523, 535 ff; Hорт/Roth, aaO (Fn. 1), $\mathbb{1} 11$ AktG Rdn. $670 \mathrm{ff}$, auch für einzelne Geschäfte Rdn. 690, Fälle Rdn $692 \mathrm{ff}$; Habersack, aaO (Fn. 1), \111 AktG Rdn. 114, 144; Spindler, aaO (Fn. 1), \111 AktG Rdn. 61 ff, 63, 67.

67 OLG Frankfurt NZG 2015, 514, 515; weitere Nachweise bei Lieder, ZGR 2018, 523, $550 \mathrm{ff}$.

68 OLG Stuttgart NZG 2002, 971, 972; OLG Frankfurt NZG 2015, 514, 515; weitere Nachweise bei LIEDER, ZGR 2018, 523, $550 \mathrm{ff}$.

69 Statt mancher Habersack, aaO (Fn. 1), $\ 116$ AktG Rdn. 43 m. w. N. zu beiden Meinungen.

70 Hopt/Roth, aaO (Fn. 1), $₫ 111$ AktG Rdn. 326.

71 Nach $₫ 87$ Abs. 2 AktG. Einzelnachweise bei Lieder, ZGR 2018, 523, $545 \mathrm{ff}$.

72 BGHZ 207, 190 Rdn. 45. 
Entscheidung über die Herabsetzung. Die unterschiedliche Behandlung beider Fälle ist in der Tat fragwürdig, doch brauchen die Argumente für und wider hier nicht näher abgewogen zu werden. Für die vorliegenden Zwecke kommt es darauf an zu sehen, dass der Aufsichtsrat auf jeden Fall eine wichtige Aufgabe hat, sei sie voll unternehmerischer Art, sei sie streng sanktionierender Art.

\section{c) Grenzstreit: Der Investorendialog des Aufsichtsrats}

Die Unsicherheiten bei der tatsächlichen Grenzziehung, was die Aufgaben und Befugnisse des Aufsichtsrats angeht, zeigt ein weiteres, hoch aktuelles Beispiel: der geradezu erbitterte Streit um den Investorendialog des Aufsichtsrats. Hierzu hat sich der Deutsche Corporate Governance Kodex 2017 auf eine Anregung dahingehend festgelegt, dass der Aufsichtsratsvorsitzende in angemessenem Rahmen bereit sein sollte, mit Investoren über aufsichtsratsspezifische Themen Gespräche zu führen, ${ }^{73}$ nachdem die ursprünglich angedachte Empfehlung auf Widerstand gestoßen war. Zuvor hatte eine Arbeitsgruppe „Developing Shareholder Communication ", an der ich von akademischer Seite beteiligt war, Leitsätze für einen solchen Dialog entwickelt ${ }^{74}$ : Der Aufsichtsratsvorsitzende entscheidet, so die Leitsätze, ob ein solcher Dialog geführt wird, und vertritt den Aufsichtsrat in der Kommunikation mit den Investoren, wobei er andere Aufsichtsratsmitglieder, den Vorstandsvorsitzenden oder andere Vorstandsmitglieder zu dem Dialog hinzuziehen kann. Themen des Dialogs können sein: Zusammensetzung und Vergütung des Aufsichtsrats, Binnenorganisation und Überwachungsprozess, Vorstandsbestellung, -abberufung und -vergütung, Strategieentwicklung und -umsetzung sowie Abschlussprüfer. Wichtig dazu ist Leitsatz 8: Ausgestaltung: Der Aufsichtsrat bespricht mit dem Vorstand Grundsätze für die Ausgestaltung eines Dialogs zwischen Investor und Aufsichtsrat. Schon zuvor war ein solcher Dialog im Schrifttum beobachtet und auch positiv beurteilt worden, ${ }^{75}$ andere lehnen ihn ab. ${ }^{76}$ Tatsächlich ist der Investorendialog nicht nur mit dem Vorstand, sondern auch mit dem Aufsichtsrat inzwischen je-

73 DCGK vom 17.2.2017 Ziff. 5.2 (2); unverändert im DCGK-E vom 6.11.2018 Empfehlung A.2. Ursprünglich hatte die DCGK-Kommission eine Empfehlung vorgeschlagen, dagegen DAV-HandelsRechtsausschuss, NZG 2017, $5759 \mathrm{f}$.

74 Leitsätze für den Dialog zwischen Investor und Aufsichtsrat, AG 2016, R 300. Dazu ausführlich mit rechtsdogmatischer, rechtspolitischer und rechtsvergleichender Begründung Hirt/Hopt/Mattheus, AG 2016, 725.

75 Z.B. Fleischer/Bauer/Wansleben, DB 2015, 360; auch schon Roth, ZGR 2012, 343, $368 \mathrm{f}$.

76 Z.B. E. Vetter, AG 2014, 387. Nachweise zu beiden Meinungen bei Hirt/Hopt/MatTHEUs, AG 2016, 725, 730. 
denfalls bei den deutschen DAX-30-Gesellschaften eine gängige Praxis. ${ }^{77}$ Die Reaktion im juristischen Schrifttum gegen die Anregung im Deutschen Corporate Governance Kodex und die Leitsätze der Arbeitsgruppe waren erstaunlich heftig, wie etwa die rhetorische Frage der Kritik zeigt: „Ist das deutsche Aktiengesetz ,lebensfremd ““78. Schwierige Grenzlinien verlaufen, wie zu den Leitsätzen ohne weiteres zuzugeben und konkretisiert und auch international diskutiert, ${ }^{79}$ beim Verbot der Weitergabe von Insiderinformationen und dem Gebot der Ad-hoc-Publizität, bei der aktienrechtlichen Verschwiegenheitspflicht und vor allem bei der Gleichbehandlung der Aktionäre, die namentlich etwa in den USA, dem Vereinigten Königreich und Belgien auch kapitalmarktrechtlich durchaus ernst genommen wird ${ }^{80}$. Dass alle drei Grenzlinien in genau derselben Weise für den offenbar akzeptierten Investorendialog des Vorstands gelten, kam in der Kritik allerdings weniger zum Vorschein. Auf Widerstand stieß insbesondere die Einbeziehung der Strategieentwicklung und -umsetzung in den Dialog, dies obschon der Kodex zu Recht vorsieht, dass der Vorstand die strategische Ausrichtung des Unternehmens mit dem Aufsichtsrat abstimmt und mit ihm in regelmäßigen Abständen den Stand der Strategieumsetzung erörtert. ${ }^{81}$ Dass von Gewerkschaftsseite im Hinblick auf die Arbeitnehmermitbestimmung die Rolle des Aufsichtsratsvorsitzenden in Frage gestellt wurde, war hingegen zu erwarten. Insgesamt war der maßgebliche Einwand ein dogmatischer: ${ }^{22}$ Eine Durchbrechung der $\$ \int 77,111$ Abs. 4 Satz $1 \mathrm{AktG}$-also die Geschäftsführung des Vorstands und das Verbot der Übertragung der Geschäftsführung an den Aufsichtsrat - durch Zulassung eines institutionalisierten Dialogs zwischen Investor und

77 Hirt/Hopt/Mattheus, AG 2016, 725, 728: „fest etabliert und wird wohl auch in anderen deutschen Unternehmen Einzug halten."

78 So der erste Satz des maßgeblichen Gegenbeitrags von Koch, AG 2017, 129.

79 Нirt/Норт/Matтheus, AG 2016, 725, 735 ff, speziell zur Gleichbehandlung ebenda $737 \mathrm{ff}:$ Норт, The Dialogue between the Chairman of the Board with Investors, Revue Trimestrielle de Droit Financier (RTDF) 2017, no. 3, 97, 100 et seq.

80 Hорт, Revue Trimestrielle de Droit Financier (RTDF) 2017, no. 3, 97, 100 et seq. mit Nachweisen zu den Corporate Governance Codes im Vereinigten Königreich, Frankreich, den Niederlanden und Belgien und der US-amerikanischen Regulation Fair Disclosure (FD) Rule 100(a); STRAmpelLi, Knocking at the Boardroom Door: A Transatlantic Overview of Director-Institutional Investor Engagement in Law and Practice, 12 Virginia Law and Business Law Review 187 (2018), auch abrufbar unter: https://ssrn. com/abstract=3044278 (letzter Zugriff: 25.6.2019); MosCA, ECFR 2018, 805. Auch VeRSE, Der Gleichbehandlungsgrundsatz im Recht der Kapitalgesellschaften, 2006, S. $537 \mathrm{f}$. Für die USA FisH, Regulation FD: an alternative approach to addressing information asymmetry, in: Bainbridge, ed., Research Handbook on Insider Trading, 2013, p. 112.

81 DCGK 2017 Ziff. 3.2. Gleichsinnig DCGK-E 2019 Grundsatz 2. Dazu LuTTER, in: Kremer/Bachmann/Lutter/v Werder, Deutscher Corporate Governance Kodex, 7. Aufl., 2018, Rdn. 510: Nicht nur Information, sondern der Aufsichtsrat soll die strategische Planung auch mittragen.

$82 \mathrm{KocH}, \mathrm{AG} \mathrm{2017,} \mathrm{129,} \mathrm{134:} \mathrm{Investorenkontakte} \mathrm{jenseits} \mathrm{der} \mathrm{Rechtsfortbildung.}$ 
Aufsichtsrat sei de lege lata ausgeschlossen. Das Anliegen sei zwar aus Investorensicht durchaus nachvollziehbar, aber liege in den Händen des Gesetzgebers. Der Streit geht juristisch weiter, die jüngsten Kommentarmeinungen variieren, ${ }^{83}$ aber auch entschiedene Gegner de lege lata geben inzwischen zu, dass der Kodex für die Praxis Fakten geschaffen hat. ${ }^{84}$ Von Seiten der Praxis wird unter die Erfolgsfaktoren eines exzellenten Aufsichtsrats auch die Stakeholder-Kommunikation gezählt, ${ }^{85}$ dabei wird die Rolle des Aufsichtsrats in der Krisenkommunikation besonders hervorgehoben. ${ }^{86}$ International ist der Dialog, allerdings für den Verwaltungsrat, mit den oben genannten drei Grenzziehungsproblemen fest etabliert. ${ }^{87}$

\section{Aufsichtsrat und Information}

\section{a) Information als Kernfrage für die Überwachung durch den Aufsichtsrat}

Allgemein anerkannt ist heute, dass die Information für den Aufsichtsrat zentral ist. Ohne zuverlässige Information über das Geschehen in der Aktiengesellschaft kann er weder effektiv überwachen noch sinnvoll beraten. Wichtigste Informationsquelle ist herkömmlich der Vorstand mit seinen Berichten

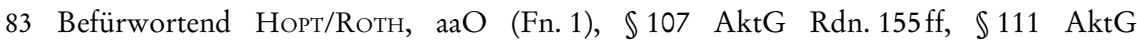
Rdn. $577 \mathrm{ff}, \mathbb{} 116$ AktG Rdn. 226; Kremer, in: Kremer/Bachmann/Lutter/v Werder, Deutscher Corporate Governance Kodex, 7. Aufl., 2018, Rdn. $1269 \mathrm{ff}$; ebenso SPIndLER, aaO (Fn. 1), $\ 107$ AktG Rdn. 40, aber in Grenzen; Bachmann, in: Gesellschaftsrechtliche Vereinigung VGR 2016, 2017, S. 135; Rотн, FS Bergmann, 2018, S. 565; wichtig und praktiziert namentlich in der Unternehmenskrise, SCHilha/Theusinger, NZG 2019, 521. Dagegen Koch, AG 2017, 129; Koch, aaO (Fn. 1), \$111 AktG Rdn. 35. Nur in den Grenzen der eigenen Zuständigkeit Habersack, aaO (Fn. 1), 116 AktG Rdn. 67; DERs, in: Habersack/Henssler, Mitbestimmungsrecht, 4. Aufl., 2018, $\$ 25$ MitbestG Rdn. 112a. Zusammenfassend auch Reutershahn, Unternehmensbezogene Außenkommunikation des Aufsichtsratsvorsitzenden, 2019; Sсніск, WPg 2018, 1379. Für die Schweiz Daeniker, FS von der Crone, 2017, S. 535.

84 E. Vetter, NZG 2018, 899: Der Kodex hat für die Rechtspraxis Fakten geschaffen; KreMER, aaO (Fn. 83), Rdn. 1269c: ganz überwiegend anerkannt. Auch Schilha/TheusinGER, NZG 2019, 521, 527: ,in der Praxis an- und aufgenommen“.

85 v. Werder, DB 2017, 997, 983. Allgemeiner Dedeyan, Regulierung der Unternehmenskommunikation, 2015.

86 Nörr/Hering Schuppener Consulting/v. Werder, Die Rolle des Aufsichtsrats in der Krisenkommunikation, 2018.

87 Aus internationaler Sicht HiRT, in: Schoppen, Corporate Governance, 2015, S. 234, 248 ff; Strampelli, aaO (Fn. 80). Der UK High Court hat in Stobart Group v. Tinkler, 2019 EWHC 258, gerade entschieden, dass Aktionäre, die im board sitzen, nicht ohne Genehmigung des board Informationen nach außen geben und in ihrer Funktion als Aufsichtsratsmitglieder andere Aktionäre und Angestellte im Hinblick auf einen battle for corporate control kontaktieren dürfen. 
über die beabsichtigte Geschäftspolitik und andere grundsätzliche Fragen der Unternehmensplanung, die Rentabilität der Gesellschaft, den Gang der Geschäfte und die Geschäfte, die für die Rentabilität oder Liquidität der Gesellschaft von erheblicher Bedeutung sein können. Der diesbezügliche $\ 90 \mathrm{AktG}$ ist 1998 grundlegend verstärkt worden. Der Aufsichtsrat und jedes einzelne Aufsichtsratsmitglied können weitere erforderliche Informationen verlangen, die letzteren allerdings nur an den Aufsichtsrat ( $\mathbb{S} 90$ Abs. 3 AktG). Hinzu kommen Rechnungslegungsunterlagen, der Abhängigkeitsbericht und der Abschlussprüferbericht sowie ein umfassendes Einsichts- und Prüfungsrecht des Aufsichtsrats im Rahmen seiner Überwachungsaufgabe ( $\mathbb{1 1 1}$ Abs. $2 \mathrm{AktG}){ }_{.}^{88}$

\section{b) Information als Voraussetzung für die business judgment rule}

Bei den unternehmerischen Entscheidungen des Aufsichtsrats und dem ihm dabei zukommenden unternehmerischen Ermessen kommt es entscheidend auf die Information an. Tatbestandliche Voraussetzung der business judgment rule ist, dass das Organmitglied auf der Grundlage angemessener Information handelt. ${ }^{89}$ Auch wenn der Bundesgerichtshof dazu klar überzogen verlangt, „,in der konkreten Entscheidungssituation alle verfügbaren Informationsquellen tatsächlicher und rechtlicher Art" auszuschöpfen, ${ }^{90}$ sind die Anforderungen dabei hoch. Die Rechtsprechung lehnt einen unternehmerischen Entscheidungsspielraum hinsichtlich der Angemessenheit der Information ab und räumt nur einen Beurteilungsspielraum ein, insoweit nämlich das Organmitglied bei einer unternehmerischen Entscheidung „vernünftigerweise annehmen durfte", auf der Grundlage angemessener Information zum Wohle der Gesellschaft zu handeln. ${ }^{91}$ Dabei kann der Aufsichtsrat, soweit ihm die eigene Sachkunde fehlt, externe Beratung einholen und kann dazu im Einzelfall sogar verpflichtet sein. ${ }^{92}$

$88 \mathrm{Zu}$ den Informationsmöglichkeiten des Aufsichtsrats Schraud, Compliance in der Aktiengesellschaft, 2019, S. $117 \mathrm{ff}, 225 \mathrm{f}$. Zur Information des Aufsichtsrats umfassend im Vergleich mit dem englischen board LeYens, aaO (Fn. 44).

$89 \int \mathbb{S} 116$ Satz 1 mit $\$ 93$ Abs. 1 Satz 2 AktG; ausführlich zur business judgment rule beim Aufsichtsrat Норт/Rотн, aaO (Fn. 1), $\$ 116$ AktG Rdn. 59-71.

90 BGH ZIP 2008, 1675 Rdn. 11 (zur GmbH); auch BGHZ 197, 304 Rdn. 30; BGHSt NJW 2017, 578 Rdn. 34 (HSH-Nordbank). Kritisch dazu Hорт/Rотн, aaO (Fn. 1), \} 9 3 AktG Rdn. 105; ebenso, „überzogen“, Lieder, ZGR 2018, 523, 555 f; auch Kubiciel, JZ 2017, 585.

91 Dazu Lieder, ZGR 2018, 523, $554 \mathrm{ff}$.

92 Lieder, ZGR 2018, 523, $564 \mathrm{ff}$. 
c) Grenzstreit: Die vorstandsunabhängige Information

Wie zuvor bei dem Investorendialog ist auch hier die tatsächliche Grenzziehung streitig. Die bisher wohl noch ü.L. spricht sich gegen ein Recht des Aufsichtsrats aus, am Vorstand vorbei die Belegschaft zu befragen, außer etwa bei Verdacht eines pflichtwidrigen Verhaltens des Vorstands oder bei Beeinträchtigung des gegenseitigen Vertrauensverhältnisses. ${ }^{93}$ Der Mindermeinung werden teilweise ein Widerspruch zum aktienrechtlichen Informationssystem, die Inkaufnahme eines Autoritätsverlusts des Vorstands und die Beeinträchtigung der Funktions- und Handlungsfähigkeit des Unternehmensleitungsorgans unterstellt. ${ }^{94}$ Von alledem kann keine Rede sein. ${ }^{95}$ Gewiss ist die Vorstandsinformation das klassische Leitbild. ${ }^{96}$ Aber die gesetzliche Regelung zur Aufsichtsratsinformation ist anders als die Information durch den Abschlussprüfer offen, und der Aufsichtsrat wird und muss selbstverständlich darauf achten, dass er den Vorstand nicht beschädigt, das ist eine Frage der Vernunft und der Ausgestaltung der Aufsichtsratskontrolle. Aber es gilt die Überwachung des Vorstands zu effektuieren. ${ }^{97}$ Damit ist auf jeden Fall unvereinbar, wenn man die Befragung von Angestellten nicht nur in gewichtigen Ausnahmefällen - darüber lässt sich reden -, sondern auch erst nach erfolglosem Hinzuziehen des Vorstands verlangt. ${ }^{98}$ Der Bundesgerichtshof hat deshalb in einer Entscheidung von 2018 zu Recht ausgeführt: „Die Notwendigkeit einer vorstandsunabhängigen Information des Aufsichtsrats folgt unmittelbar aus seiner Aufgabe, den Vorstand eigenverantwortlich zu überwachen, da ansonsten der zu

93 Statt vieler Koch, aaO (Fn. 1) $\ 111$ AktG Rdn. 21 mit umfangreichen Nachweisen zum Meinungsstreit.

94 So anders als sonst im Aufsatz verkürzt Lieder, ZGR 2018, 523, 564, aber in der Sache mit den Argumenten der ü.L. Dass es zum Konflikt zwischen zwei Alphatieren kommen kann, ist richtig, aber ein Problem, das sich nicht erst und nicht nur bei der vorstandsunabhängigen Information stellt.

95 Für vorstandsunabhängige Information ausführlich Норт/Rотн, aаO (Fn. 1), \111 AktG Rdn. 480-517; Habersack, aaO (Fn. 1), \109 AktG Rdn. 19, 1111 AktG Rdn. 80; auch Schraud, aaO (Fn. 88), S. 124 ff; zur SE SCHAPER, AG 2018, 356. Ebenso für den österreichischen Aufsichtsrat Kalss, aaO (Fn. 1), $\$ 95$ AktG Rdn. 13: jedenfalls einmal pro Jahr, sonst bei begründeten Anlassfällen. Informationen erhält der Aufsichtsrat auch mittelbar aus dem Betriebsrat über die Arbeitnehmervertreter im Aufsichtsrat.

$96 \rrbracket 90$ AktG Berichte (des Vorstands) an den Aufsichtsrat, mit Änderungen 1998, 2002 und 2015.

97 Zutr. Habersack, aaO (Fn. 1), $\mathbb{1} 111$ AktG Rdn. 80; Roth, ZGR 2012, 343, 372 ff. Zu einer Durchsuchung der E-Mails von Vorstandsvorsitzenden im Konzern auf Rechtsrisiken (Deutsche Bank), Nienhaus, Die ZEIT Nr. 18, 25.4.2019, S. 19.

98 So aber Spindler, aaO (Fn. 1), $\$ 111$ AktG Rdn. 36. 
kontrollierende Vorstand über die Auswahl der mitgeteilten Informationen seine Überwachung faktisch ausschalten könnte." ${ }^{\text {"99 }}$

Die rechtlich und praktisch eigentlichen Fragen bei der vorstandsunabhängigen Informationsbeschaffung liegen denn auch nicht bei dem theoretisch bleibenden Extremfall, dass der Aufsichtsrat am Vorstand vorbei willkürlich auf irgendwelche Angestellte im Unternehmen zugeht. Vielmehr geht es zum einen darum, ob der Aufsichtsratsvorsitzende und eventuell auch der Prüfungsausschusses direkt mit den Leitern der internen Kontrollinstanzen, also dem Leiter der internen Revision, dem Chief Compliance Officer (CCO, Leiter der Compliance) und dem Leiter des Risikomanagements (CRO, Chief Risk Officer) kommunizieren darf. ${ }^{100}$ Wenn diese Personen sich allein an den Vorstand halten könnten und umgekehrt der Aufsichtsrat sie nur indirekt über den Vorstand befragen könnte bzw. einen konkreten Verdacht gegen den Vorstand abwarten müsste, wäre eine wirksame Überwachung durch den Aufsichtsrat oder einen von ihm eingesetzten Ausschuss nach $\$ 107 \mathrm{Abs}$. 3 Satz 2 AktG ${ }^{101}$ nicht garantiert. Zum anderen stellt sich die Frage nach einem Zugriff des Aufsichtsrats und für diesen des Aufsichtsratsvorsitzenden auf die internen Kontrollsysteme der Gesellschaft. ${ }^{102}$ Insgesamt empfiehlt sich eine Regelung dieser Fragen gemeinsam durch Vorstand und Aufsichtsrat, am besten im Rahmen

BGH, 20.3.2018, AG 2018, 436 Rdn. 21. Der anschließende Satz, in dem der BGH die Befugnis des Aufsichtsrat erwähnt, Sachverständige heranzuziehen ( $\$ 109$ Abs. 1 Satz 2 AktG) oder sie für bestimmte Prüfungsaufgaben zu beauftragen ( $\$ 111$ Abs. 2 Satz 2 $\mathrm{AktG}$ ), ist nicht als Einschränkung der vorstandsunabhängigen Information auf diese beiden Fälle zu verstehen, sondern erklärt sich aus der zu entscheidenden Rechtsfrage nach der prozessualen Vertretung der Gesellschaft gegenüber einem vom Aufsichtsrat beauftragten Sachverständigen.

100 Für Kreditinstitute gilt schon heute nach $\ 25 \mathrm{~d}$ Abs. 8 Satz 7, 8, Abs. 9 Satz 4, 5 und Abs. 12 Satz 7, 8 KWG, dass die Vorsitzenden des Risikoausschusses, des Prüfungsausschusses und des Vergütungskontrollausschusses oder, falls ein solcher Ausschuss nicht eingerichtet wurde, der Vorsitzende des Verwaltungs- oder Aufsichtsorgans unmittelbar beim Leiter der Internen Revision und beim Leiter des Risikocontrollings bzw. bei den Leitern der für die Ausgestaltung der Vergütungssysteme zuständigen Organisationseinheiten Auskünfte einholen können. Dass dies nicht einfach per Analogie oder „Ausstrahlung“ eins zu eins in das Aktienrecht übertragen werden kann, sei zur Vermeidung von Missverständnissen klargestellt. Zur eigenständigen Corporate Governance von Banken Hopt, ZGR 2017, 438 mit umfassenden Nachweisen; auch schon IDEM, Journal of Corporate Law Studies 13 Part 2 (2013) 219. Auch BINDER/Glos/RIEPE, Handbuch Bankenaufsichtsrecht, 2018, \11 III: Organe und Organmitglieder.

$101 \$ 107$ Abs. 3 Satz 2 AktG: Insbesondere ein Prüfungsausschuss, „der sich mit der Überwachung des Rechnungslegungsprozesses, der Wirksamkeit des internen Kontrollsystems, des Risikomanagementsystems und des internen Revisionssystems sowie der Abschlussprüfung ... befasst“. Zum Prüfungsausschuss als Empfänger vorstandsunabhängiger Information Hорт/Rотн, aaO (Fn. 1), $\$ 111$ AktG Rdn. 510.

102 Hopt/Roth, aaO (Fn. 1), $\$ 111$ AktG Rdn. 487 ff. 
einer allgemeinen Informationsordnung, ${ }^{103}$ so wie schon für die oben erörterte andere Grenzziehungsproblematik des Investorendialogs des Aufsichtsrats. ${ }^{104}$ Für Kreditinstitute gilt schon heute nach $\$ 25 \mathrm{~d}$ Abs. 8 Satz 7, 8, Abs. 9 Satz 4, 5 und Abs. 12 Satz 7, 8 KWG, dass die Vorsitzenden des Risikoausschusses, des Prüfungsausschusses und des Vergütungskontrollausschusses oder, falls ein solcher Ausschuss nicht eingerichtet wurde, der Vorsitzende des Verwaltungs- oder Aufsichtsorgans unmittelbar beim Leiter der Internen Revision und beim Leiter des Risikocontrollings bzw. bei den Leitern der für die Ausgestaltung der Vergütungssysteme zuständigen Organisationseinheiten Auskünfte einholen kann, die Geschäftsleitung hierüber aber unterrichtet werden muss. Diese Vorschriften sind nicht analogiefähige Sonderregeln, aber doch richtungsweisend.

\section{Aufsichtsrat und Interessenkonflikte}

\section{a) Interessenkonflikte des Aufsichtsrats als Nebenamt}

Mit den Interessenkonflikten des Aufsichtsrats sei noch ein weiterer Beleg dafür gebracht, dass der Aufsichtsrat in neuerer Zeit ganz erheblich an Aufmerksamkeit gewonnen hat. Aber auch hier wird sich zeigen, dass die Umbrüche zu Unsicherheiten und Streit bei den Grenzziehungen führen. Interessenkonflikte gibt es zwar auch im Verwaltungsrat, ${ }^{105}$ aber im Aufsichtsrat sind sie noch ein Stück gravierender. Das liegt daran, dass der Aufsichtsrat anders als der board oder Verwaltungsrat als Nebenamt konzipiert ist, ${ }^{106}$ auch wenn neuerdings vor allem beim Aufsichtsratsvorsitzenden, aber auch bei einfachen Aufsichtsratsmitgliedern ein Trend zum Berufsaufsichtsrat festzustellen ist. Als Grundsatz für interessenkonfliktbehaftete Aufsichtsratsmitglieder gilt, dass bei ihrem Tätigwerden als Organ das Interesse der Gesellschaft Vorrang vor Eigen- und Drittinteressen haben muss (Prioritätsgrundsatz), und dass bei Tätigwerden außerhalb der Organfunktion die eigenen Interessen zwar verfolgt werden dürfen, aber beschränkt durch eine allgemeine Rücksichtspflicht gegenüber der Gesellschaft. Konsequenz des bloßen Nebenamts ist, dass die Tätigkeit in Konkurrenzunternehmen nicht verboten ist und dass Doppel- und Mehrfachmandate zulässig bleiben. ${ }^{107}$

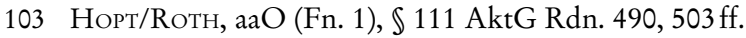

104 Oben III. 1.c).

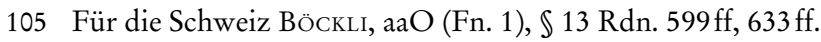

106 Hierzu und zum Folgenden Hорт/Rotн, aaO (Fn. 1), $\$ 116$ AktG Rdn. $142 \mathrm{ff}$.

107 Hорт/Rотн, aаO (Fn. 1), \$116 AktG Rdn. 151, 173. Immerhin empfiehlt Ziffer 5.4.2 Satz 4 DCGK, dass Aufsichtsratsmitglieder keine Organfunktion oder Beratungsaufgaben bei wesentlichen Wettbewerbern des Unternehmen ausüben; erweiternd DCGK-E 2019 Empfehlung C.12. 
Es liegt auf der Hand, dass damit schwierige Abgrenzungen notwendig werden. ${ }^{108}$ Das für unser Thema Entscheidende ist aber etwas anderes, nämlich dass das deutsche Recht, aber auch das österreichische und das schweizerische, ${ }^{109}$ anders als insbesondere das US-amerikanische den Interessenkonflikten herkömmlich keine besondere Aufmerksamkeit geschenkt haben. Fiduciary duties, duties of loyalty und duty of fair dealing hatten herkömmlich keine Entsprechung im deutschen Recht. Das zeigt ein Blick in die Principles of Corporate Governance des American Law Institute, wenn dort lang etablierte Interessenkonflikte für die Mitglieder des board behandelt werden: Geschäfte von Organen mit der Gesellschaft, Fragen ihrer Vergütung, Gebrauch von Gesellschaftsvermögen für eigene Zwecke, Ausnutzung von Geschäftschancen, Wettbewerb mit der Gesellschaft, Verhalten bei Kontrolltransaktionen und Übernahmeangeboten. ${ }^{110}$ Teilweise zeigt sich das noch in den „deutschen“ Begriffen wie corporate opportunities, Insidergeschäfte, $\mathrm{MBO}$, audit committee, business judgment rule u. a. Was davon legal transplants ${ }^{111}$ im eigentlichen Sinne sind, mag hier dahinstehen. Sicher ist jedenfalls, dass, wie Jan von Hein in seiner großen Hamburger Habilitationsschrift 2008 im Einzelnen dargestellt hat, US-amerikanisches Gesellschaftsrecht in Deutschland in breiter Front rezipiert worden ist. ${ }^{12}$

Schon bei der großen Reform des KonTraG von 1998 wurde die Frage der Interessenkonflikte des Aufsichtsrats breit diskutiert, ${ }^{113}$ interessanterweise mehr als die des Vorstands, was eine missverständliche Gegenüberstellung von board und Aufsichtsrat implizierte. Bei der Einführung der business judgment rule in $\ 93$ Abs. 1 Satz 2 AktG 2005 setzte dann aber die Regelung umgekehrt beim Vorstand an, wenn diesem der safe haven versagt wurde, wenn er nicht ohne Interessenkonflikte und sachfremde Einflüsse gehandelt hat. ${ }^{114}$ Diese Kodifizierung des business judgment rule und der Interessenkonflikte als negatives Tatbestandsmerkmal gilt über die Verweisung in $\$ 116$ Satz $1 \mathrm{AktG}$ auch für die Verantwortlichkeit der Aufsichtsratsmitglieder. Damit ist die Freiheit

108 Fallgruppenkatalog bei Норт/Rотн, aaO (Fn. 1), $₫ 116$ AktG Rdn. 142 ff.

109 Für die Schweiz BöckLI, aaO (Fn. 1), \13 Rdn. 665: Osmose angelsächsischen Rechts, die Treuepflicht für Verwaltungsräte, obschon anerkannt, stand bis 1991 nicht ausdrücklich im Gesetz, $\mathbb{1 3}$ Rdn. 596; zur Treupflicht nach Art. 717 OR auch BüHLER, $\mathrm{aaO}$ (Fn. 33), Art. 717 N $109 \mathrm{ff}$.

110 American Law Institute (ALI), Principles of Corporate Governance: Analysis and Recommendations, Vol. 1, St. Paul 1994, $\$ \$ 5.02$ et seq.; dazu Hopt, FS Mestmäcker, 1996, S. $909,921 \mathrm{ff}$.

111 Zuletzt Langenbucher, Economic Transplants, On Lawmaking for Corporations and Capital Markets, Cambridge 2017.

112 von HeIn, aaO (Fn. 16), S. $376 \mathrm{ff}$.

113 Nachweise von Hein, aaO (Fn. 16), S. 237 Fn. 1254.

114 Норт/Roth, aaO (Fn. 1), $\$ 93$ AktG Rdn. 90 ff. 
von Interessenkonflikten auch kodifikatorisch bei den Aufsichtsratsmitgliedern angekommen. ${ }^{15}$

\section{b) Independent directors im Aufsichtsrat und die Schlüsselrolle des Aufsichtsrats bei Übernahmen}

Zum Bedeutungszuwachs des Aufsichtsrats auch unabhängig vom Vorstand sind beispielhaft noch zwei weitere Entwicklungen anzusprechen: das Aufkommen der independent directors und die Schlüsselrolle, die das WpÜG dem Aufsichtsrat bei Übernahmen eingeräumt hat. Mit beiden fokussierte sich die Aufmerksamkeit in Diskussion und Normierung auf den Aufsichtsrat.

Das internationale Aufkommen der independent directors in den USA und dem Vereinigten Königreich und die Ausstrahlung der Idee und ihrer Kodifikation auf Deutschland sind bekannt und brauchen hier nicht nachgezeichnet $\mathrm{zu}$ werden. ${ }^{116}$ Bemerkenswert ist aber, wie zögerlich die Forderung nach independent directors in Deutschland aufgenommen wurde. Im Aktiengesetz gibt es keine Definition der Unabhängigkeit für Aufsichtsratsmitglieder, und es gibt nur spärliche Regelungen dazu. ${ }^{117}$ Immerhin hatte der alte $\$ 100$ Abs. 5 AktG vorgesehen, dass in kapitalmarktorientierten Aktiengesellschaften mindestens ein unabhängiges Aufsichtsratsmitglied über Sachverstand auf den Gebieten der Rechnungslegung oder der Abschlussprüfung verfügen muss. Die Abschlussprüferreform von 2016 hat dieses Unabhängigkeitserfordernis jedoch wieder beseitigt, was kritisiert worden ist. ${ }^{118}$ Die Unabhängigkeit von Aufsichtsratsmitgliedern bleibt deshalb den nicht bindenden Empfehlungen des Deutschen Corporate Governance Kodex vorbehalten. Aber auch dort

$115 \mathrm{Zu}$ den Interessenkonflikten der Aufsichtsratsmitglieder bei der business judgment rule LIEDER, ZGR 2018, 523, $568 \mathrm{ff}$.

116 Zusammenfassend zuletzt Bainbridge, aaO (Fn. 53), ch. 12, p. 309 et seq.; Hopt/Roth, aaO (Fn. 1), $\ 100$ AktG Rdn. 147 ff. Dazu rechtsvergleichend Hopt, ZHR 175 (2011), 444, 484 ff, und Rотн, ZHR 175 (2011), 605, jeweils m.w. N.

$117 \mathrm{Zu}$ den Interessenkonflikten im Aufsichtsrat ausführlich SеIвт, FS Hopt, Bd. 1, 2010, S. 1363. Auch von Hein, aaO (Fn. 16), S. 895 ff. Aus den Kommentaren Hopt/Roth,

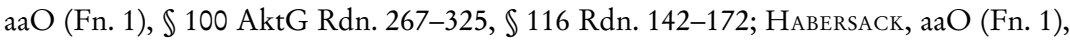
$\$ 100$ AktG Rdn. 92 ff; SpIndler, aaO (Fn. 1), $\$ 116$ AktG Rdn. 84 ff; kurz auch Koch, $\mathrm{aaO}$ (Fn. 1), $\mathbb{} 108 \mathrm{AktG}$ Rdn. $10 \mathrm{ff}$. Zu Interessenwahrung und Interessenkonflikten im Aktien-, Bank- und Berufsrecht Hopt, ZGR 2004, 1 ff. Zu Prävention und Repression von Interessenkonflikten im Aktien-, Bank- und Berufsrecht DERS., FS Doralt,

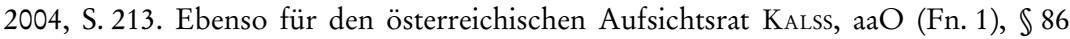
AktG Rdn. 66ff; Frotz/SchÖrghofer, in: Kalss/Kunz, Handbuch für den Aufsichts-

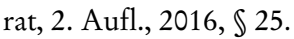

118 Nodouschani, AG 2016, 381, 383 f; befürwortend dagegen Schilha, ZIP 2016, 1316, $1317 \mathrm{ff}$. International schwer vermittelbar, aber derzeit zu akzeptieren, Hорт/Rотн, aaO (Fn. 1), $\$ 100$ AktG Rdn. 236. Ohne Stellungnahme Kremer, aaO (Fn. 83), Rdn. 1374. 
verlief die Diskussion harsch und die Erfolge blieben hinter der internationalen Entwicklung zurück. Das ist wesentlich eine Folge der pfadabhängigen deutschen Mitbestimmung im Aufsichtsrat. Schon für die Kommission, die den Deutschen Corporate Governance Kodex erarbeitet hat, war die Mitbestimmung ein Tabu, ${ }^{119}$ und dass die Arbeitnehmer im Aufsichtsrat nicht unabhängig im Sinne der Corporate Governance sind, wird noch heute von einer breiten Meinung, nicht nur von Seiten der Gewerkschaften, bestritten. ${ }^{120}$ Für die Anteilseignerseite im Aufsichtsrat sparte der Kodex zunächst die Unabhängigkeit von beherrschenden Aktionären aus und verzichtete bewusst auf eine positive oder negative Definition der Unabhängigkeit. Erst der Entwurf für eine Kodexreform von 2018 beinhaltete eine Definition und eine ausführliche Liste von Kriterien für die Einschätzung der Unabhängigkeit seiner Mitglieder durch den Aufsichtsrat. ${ }^{121}$ Entscheidend ist danach, dass dem Aufsichtsrat auf Anteilseignerseite „eine nach deren Einschätzung angemessene Zahl unabhängiger Mitglieder" angehört, wobei das pflichtgemäße Ermessen der Anteilseignerseite sich nicht nur auf die Angemessenheit, sondern auch auf die Unabhängigkeit erstreckt, bei Bejahung der Unabhängigkeit entgegen den Kriterien mit Begründungsempfehlung. ${ }^{122}$

Einen erheblichen Bedeutungszuwachs erhielt der Aufsichtsrat durch die Rolle, die ihm der Gesetzgeber des WpÜG 2001 zuwies. $\ 33$ Abs. 1 Satz 1 WpÜG normiert in Übereinstimmung mit der Europäischen Übernahmerichtlinie das Neutralitätsgebot bzw. richtiger das Verhinderungsverbot für den Vorstand. Satz 2 enthält zunächst die beiden üblichen Ausnahmen: Handlungen eines ordentlichen und gewissenhaften Geschäftsleiters einer Gesellschaft, die nicht von einem Übernahmeangebot betroffen ist, und Suche nach einem konkurrierenden Angebot. Dann folgt jedoch in Satz 2 ganz unauffällig eine dritte Ausnahme: „sowie für Handlungen, denen der Aufsichtsrat der Zielgesellschaft zugestimmt hat“. Während also etwa im Vereinigten Königreich das Verhinderungsverbot für den gesamten board gilt, ist der im zweistufigen System abgespaltene Aufsichtsrat Herr der Verteidigungsmaßnahmen. Das ist als Sündenfall und Schaden für den Finanzplatz kritisiert wor-

119 Angeblich soll das Aussparen der Mitbestimmung eine Vorgabe von Regierungsseite bei der Aufgabenbetrauung der Baums-Kommission gewesen sein. Das war schon damals und ist erst recht heute nicht überzeugend, weder in der Sache noch erst recht im Hinblick auf die in Anspruch genommene Informationsfunktion des DCGK für ausländische Investoren.

120 Dazu Roth, ZHR 175 (2011), 605, $638 \mathrm{ff}$.

121 DCGK-E 2018 Empfehlungen Ziffer B.7 bis B.13, Kriterienliste in B.8. Auch DCGKE 2019 Ziffer C.6-C.12, Kriterienliste C.7.

122 DCGK-E 2018 Empfehlungen Ziffer B.7 (1), B.8, B.9 mit Begründungen zu B.7-B.11. Auch DCGK-E 2019 C.6 (1), C.7, C.8 mit Begründungen zu C.6-C.8 und C.10. Zum Einschätzungsermessen des Aufsichtsrats auch Норт/Rотн, aaO (Fn. 1), $\$ 100$ AktG Rdn. $170 \mathrm{ff}$. 
den. ${ }^{123}$ Ein US-amerikanischer Beobachter sprach sogar im Vergleich selbst mit dem Recht von Delaware von einem „Anti'-Übernahmegesetz“. ${ }^{124}$ Das ist sicher überzogen. Aber das für eine gute, wirksame Corporate Governance notwendige Zusammenwirken der internen Corporate Governance, die auch in Deutschland ernst genommen wird, mit der externen Corporate Governance durch Übernahmen ${ }^{125}$ ist dadurch weitgehend ausgehebelt. Bezeichnend ist dabei, dass die Diskussion um independent directors, denen die Entscheidung im Aufsichtsrat vorbehalten werden könnte, bei Übernahmen in Deutschland weder im Gesetz noch in der Diskussion Boden gefasst hat. ${ }^{126}$ Feindliche Übernahmen kommen in Deutschland vor, aber spielen bei weitem nicht die Rolle wie in anderen Ländern. ${ }^{127}$ Es könnte aber sein, dass, wie neuestens von ökonomischer und auch juristischer Seite, bemerkt wird, die institutionellen Investoren sich anschicken, bis zu einem gewissen Grad diese Aufgabe der externen Corporate Governance zu übernehmen. ${ }^{128} \mathrm{Ob}$ das wirklich der Fall ist und wie sich das auf die Längerfristigkeit der unternehmerischen und Anlageentscheidungen auswirkt, ist eine der wichtigsten aktuellen Fragen und hoch kontrovers.

123 Hopt, ZHR 166 (2002), 383, 427; DöRING, BörsZ Nr. 218 vom 10.11.2001, 1. Zu dem Streit um das Verhinderungsverbot befürwortend Hopt, Verhaltenspflichten des Vorstands der Zielgesellschaft bei feindlichen Übernahmen, Festschrift für Lutter, 2000, S. 1361; dagegen Seıвт, in: Mülbert/Kiem/Wittig, 10 Jahre WpÜG, 2011, S. 148; dazu wiederum Норт, Europäisches Übernahmerecht, 2013, S. 69 ff und IDEм, Takeover Defenses in Europe: A Comparative, Theoretical and Policy Analysis, Columbia Journal of European Law 20 (2014) 249. Sogar verfassungsrechtlich bedenklich, KORT, Großkomm. z. AktG, 5. Aufl., 2015, \$ 76 Rdn. 140. Jedenfalls für volle richterliche Kontrolle HeYers, Der Konzern 2017, 231, 240. Abwägend Habersack, ECFR 2018, 1, 13 et s. Zu Frankreich zuletzt Segain/Devaux, Revue Trimestrielle de Droit Financier 2018, 22.

124 Gordon, AG 2002, 670. Dazu aber relativierend von Hein, aaO (Fn. 16), S. 894 f.

125 Zur Unterscheidung von interner und externer Corporate Governance Hopt, in: Hommelhoff/Hopt/v. Werder, Handbuch Corporate Governance, 2. Aufl., 2009, S. 39, $44 \mathrm{ff}$, $50 \mathrm{ff}, 57 \mathrm{ff}$; DERs., ZHR 175 (2011), 444, $450 \mathrm{ff}$. Aus den USA berichten Gisgab/HanNES, The Death of Corporate Law, New York University Law Review 94 (2019) 263, dass die institutionellen Investoren am US-Markt erfolgreich gegen poison pills, staggered boards und golden leashes und für indices excluding issuers of multiclass shares vorgehen und die board-freundlichere Rechtsprechung der Delaware courts praktisch relativieren und auf procedural supervision zurückdrängen; das als Tod des Aktienrechts zu bezeichnen, mag in den USA gut ankommen, ist aber weit überzogen.

$126 \mathrm{Zu}$ den Interessenkonflikten im Aufsichtsrat der Zielgesellschaft aber HоPт, ZGR 2002, 333; E. VetTer, FS Hopt, Bd. 2, 2010, S. 2657.

127 Z.B. Hogan Lovells, Öffentliche Übernahmen in Deutschland, Newsletter 2019.

128 Dazu Habersack, ECFR 2018, 1, 23 et seq., 28; Hell, NZG 2019, 338, 342. 
c) Grenzstreit: Related party transactions, der Aufsichtsrat und sein Ausschuss nach dem Regierungsentwurf des ARUG II

Das dritte, zeitlich jüngste Beispiel für den Bedeutungszuwachs des Aufsichtsrats ist die anstehende Kodifikation der related party transactions in Umsetzung der Aktionärsrechte-Richtlinie II (Umsetzungsfrist an sich bis 10. Juni 2019). ${ }^{129}$ Der Regierungsentwurf eines ARUG II sieht vor, dass bei einer börsennotierten Aktiengesellschaft Geschäfte mit nahestehenden Personen ${ }^{130}$ ab einen bestimmten hohen Schwellenwert ${ }^{131}$ der vorherigen Zustimmung des Aufsichtsrats bedürfen ( $(111$ b Abs. 1 AktG-E). Bei der Beschlussfassung können diejenigen Aufsichtsratsmitglieder ihr Stimmrecht nicht ausüben, die an dem Geschäft als nahestehende Personen beteiligt sind oder bei denen die Besorgnis eines Interessenkonflikts aufgrund ihrer Beziehung zu der nahestehenden Person besteht $\left(\mathbb{1 1 1}\right.$ b Abs. 2 AktG-E). ${ }^{132}$ Der Aufsichtsrat kann, aber muss nicht ${ }^{133}$ die Entscheidung über die Zustimmung an einen eigenen oder, wie die Begründung klarstellt, bestehenden Ausschuss delegieren, der darüber beschließt, allerdings ohne weiter an einen Unterausschuss delegieren zu können ${ }^{134}$. Diesem Ausschuss können nicht nur keine an dem Geschäft beteiligte nahestehende Personen angehören, sondern er muss auch mehrheitlich aus Mitgliedern zusammengesetzt sein, bei denen keine Besorgnis eines Interessenkonflikts aufgrund ihrer Beziehungen zu einer nahestehenden Person besteht ( $\$ 107$ Abs. 3 Satz 4

129 Ausführliche Nachweise zu RefE und RegE ARUG II in Deutschland, Österreich und der Schweiz aaO (Fn. 2). Die Befassung der Hauptversammlung mit der Zustimmung wäre schwerfällig, kostspielig und ganz impraktikabel, LIEDER/WERNERT, ZIP 2018, $2441,2448$.

130 \111 a Abs. 1 AktG-E mit Definition der Geschäfte und über Weiterverweisung auf IAS 24 sowie IFRS 10 und 11 sowie IAS 28 (RegE ARUG II Begründung S. 90) bezüglich der nahestehenden Personen sowie Abs. 2 mit weitreichenden Ausnahmen, allerdings nicht für den faktischen Konzern, zu Recht, J. SснміDт, EuZW 2019, 261, 262f, aber umstritten, z.B. Bungert/Berger, DB 2018, 2860, 2862; Heldt, AG 2018, 905, 915f; MÜLLER, ZGR 2019, 97, 115 ff: für Geschäfte mit Tochtergesellschaften, also nur downstream.

$131 \$ 111$ b Abs. 1 AktG-E: Schwellenwert, wenn der wirtschaftliche Wert des Geschäfts 2,5 \% der Summe aus Anlage- und Umlaufvermögen der Gesellschaft bzw. des Konzerns übersteigt. Das entspricht dem, was international üblich ist, J. Schmidt, NZG 2018, 1201, 1209 und EuZW 2019, 261, Lieder/WERnERT, ZIP 2018, 2441, 2444; auch FLEISCHER, BB 2014, 2691, $2692 \mathrm{ff}$.

132 Die konfligierten Aufsichtsratsmitglieder können also an der Beratung teilnehmen. In besonderen Fällen wird man allerdings eine Pflicht annehmen müssen, der betreffenden Aufsichtsratssitzung fernzubleiben, nämlich wenn schon die bloße Teilnahme die Interessen der Gesellschaft tatsächlich gefährdet, Норт, ZGR 2004, 1, 33 f; hohe Anforderungen, konkrete Gefährdung, Норт/Rотн, aаO (Fn. 1), $\mathbb{1 0 9}$ AktG Rdn. $21 \mathrm{ff}$ m.w.N.

133 RegE ARUG II Begründung S. 85, freies Ermessen des Aufsichtsrats.

134 RegE ARUG II Begründung S. 85. 
AktG-E). ${ }^{135}$ Gegenüber dem Referentenentwurf ist die Regelung in $\$ 107$ Abs. 3 Satz 4 bis 6 AktG auf jeden Fall weniger kompliziert und in der Sache zu Recht teils erleichternd (beschließender Ausschuss), teils verschärfend (die Besorgnis eines Interessenkonflikts bestimmt sich nicht mehr „nach Einschätzung des Aufsichtsrats", sondern ohne Ermessen desselben und ist anders als die entsprechende Beurteilung unter Nr. 5.4.2 DCGK ${ }^{136}$ gerichtlich voll nachprüfbar) ${ }^{137}$. Zwar kann der Aufsichtsrat die für einen Interessenkonflikt sprechenden Kriterien selbst präzisieren. Der Regierungsentwurf weist dazu in der Begründung zwar auf die beiden EU-Empfehlungen vom 15.2.2005 und 16.5.2002 ${ }^{138}$ hin, stellt aber ausdrücklich klar, dass der Aufsichtsrat auch bei Erfüllung der dort genannten, für einen Interessenkonflikt sprechenden Kriterien gute Gründe haben kann, anders zu entscheiden, eine schematische Anwendung der Kriterien soll nicht angezeigt sein, das sei Sache des Einzelfalls. ${ }^{139}$ Es folgen dann ausführliche, durchaus hilfreiche Hinweise und Beispiele dazu, wann bei konkreten Beziehungen insbesondere geschäftlicher, finanzieller oder persönlicher Art das Urteilsvermögen des Aufsichtsratsmitglied bei seiner Entscheidungsfindung beeinflusst werden kann, also ein Interessenkonflikt anzunehmen ist. ${ }^{140}$ Der Regierungsentwurf hat auch die (unverzügliche) Veröffentlichung, obschon der Sache nach kapitalmarktrechtlich, anders als der Referentenentwurf nicht mehr im WpHG, sondern in $\$ 111 \mathrm{c}$ AktG verortet, dabei aber zu Recht darauf geachtet, dass die schon wegen des hohen Schwellenwerts bestehenden Bezüge zur Ad-hoc-Publizität unter Art. 17 Abs. 1 UAbs. 2 MAR berücksichtigt bleiben $(\$ 111 \mathrm{c}$ Abs. $3 \mathrm{AktG}) \cdot{ }^{141}$ Das geschieht durch die Vorschrift einer einheitlichen Veröffentlichung der Insiderinformation und der related party transaction-Ver-

135 Für die Abstimmung des Ausschusses hätte es nahegelegen, $\ 111$ b Abs. 2 AktG über den Stimmrechtsausschluss auch hier heranziehen. Demgegen soll durch die Nichtanwendung ein Anreiz geschaffen werden, einen solchen Ausschuss einzurichten, in dem die Argumente aller Seiten ausgetauscht werden können, RegE ARUG II Begründung S. 85.

136 Auch dort streitig, dagegen die wohl hL; aber zu den Unterschieden der Entscheidung unter dem DCGK und zu den related party transactions beachtlich LIEDER/WERNERT, ZIP 2018, 2441, 2450.

137 RegE ARUG II Begründung S. 86, 88, keine business judgment rule, kein Beurteilungsspielraum.

138 Empfehlung der Kommission vom 15.2.2005 zu den Aufgaben von nicht geschäftsführenden Direktoren/Aufsichtsratsmitgliedern börsennotierter Gesellschaften sowie zu den Ausschüssen des Verwaltungs-/Aufsichtsrats, AblEU vom 25.2.2005, L 52/51, und vom 16.5.2002 zur Unabhängigkeit des Abschlussprüfers, AblEU vom 19.7.2002, L $191 / 22$.

139 RegE ARUG II Begründung S. 87.

140 RegE ARUG II Begründung S. $87 \mathrm{f}$.

141 RegE ARUG II Begründung S. 97 ff; J. Schmidt, EuZW 2019, 261, 264. Zu Art. 17 MAR Hopt/Kumpan, Insider- und Ad-hoc-Publizitätsprobleme, in: Schimansky/Bunte/Lwowski, Bankrechts-Handbuch, 5. Aufl., 2017, Bd. II, $\mathbb{1 0 7}$, sowie umfassend 
öffentlichung. Die Möglichkeit der Selbstbefreiung besteht hier, und zum Schutz sensibler Informationen unabhängig davon, ob eine Veröffentlichung von Insiderinformationen erforderlich ist. ${ }^{142}$

Insgesamt wird diese vorsichtige Umsetzung der Aktionärsrechterichtlinie, wie nicht anders zu erwarten und hier nicht weiter nachzuzeichnen ist, durchaus unterschiedlich beurteilt. Während die Unternehmenspraxis mit diese „minimalinvasiven " Umsetzung ${ }^{143}$ zufrieden ist und auch das aktienrechtliche Schrifttum im Ergebnis ganz überwiegend zustimmt, ist aus Investoren- und internationaler Sicht wider die „weiße Salbe“ gewettert und die Umsetzung sogar, allerdings wohl zu Unrecht, als richtlinienwidrig bezeichnet worden. ${ }^{144}$ Wichtig erscheint ein Beitrag dazu aus empirischer Sicht, der der vorgeschlagenen Regelung bestätigt, dass sie insgesamt ausgewogen und angemessen erscheint. ${ }^{145}$ Auf jeden Fall ist anzunehmen, dass diese vorsichtige Regelung, die den deutschen Sonderweg des Konzernrechts ${ }^{146}$ bewahrt, ${ }^{147}$ Ende des Jahres mit dem ARUG II Gesetz werden wird.

\section{Der zu zablende Preis: Mebr Pflichten und strengere zivil- und strafrechtliche Haftung von Aufsichtsratsmitgliedern}

Der Bedeutungswandel des Aufsichtsrats hat seinen Preis in einem ganz erheblichen Zuwachs von Pflichten und Haftungsrisiken des Aufsichtsrats, worauf zuletzt die Begründung des Regierungsentwurfs zum ARUG II ausdrücklich hingewiesen hat. Die Beurteilung, ob bei einer related party transaction ein Interessenkonflikt vorliegt, ist Aufgabe des Aufsichtsrats, aber diese Beurteilung ist gerichtlich voll überprüfbar, und bei einer Fehlbeurteilung können sich die Aufsichtsratsmitglieder schadensersatzpflichtig machen ( $\mathbb{S} 116$ Satz 1, 93 Abs. 2 AktG). ${ }^{148}$ Die Begründung des Regierungsentwurfs unterstreicht das:

KLÖHn, Marktmissbrauchsverordnung, 2018; MeYer/Veil/Rönnau, Handbuch zum Marktmissbrauchsrecht, 2018.

142 RegE ARUG II Begründung S. 99.

143 So insgesamt der Tenor der oben Fn. 2 aufgeführten Stellungnahmen.

144 Tröger/Roth/Strenger, BB 2018, 2946 zum RefE.

145 ENGERT/FloRstedt, ZIP 2019, 493, 503 zum RefE.

146 Vgl. Hopt, Groups of Companies: A Comparative Study of the Economics, Law, and Regulation of Corporate Groups, in: Gordon/Ringe, The Oxford Handbook of Corporate Law and Governance, 2018, p. 603; auch abrufbar unter: https://papers.ssrn. com/sol3/papers.cfm?abstract_id=2560935 (letzter Zugriff: 4.6.2019). Zum Vergleich des US-amerikanischen und deutschen Konzernrechts grundlegend Mestmäcker, Verwaltung, Konzerngewalt und Rechte der Aktionäre, 1958.

147 Zutr. J. SснміDт, EuZW 2019, 261, 264 a.E.: ,insgesamt sinnvolle Ergänzung des deutschen konzernrechtlichen Schutzsystems und Corporote Governance-Rahmens“.

148 RegE ARUG II Begründung S. 88, 96. 
„Dies stellt angesichts der Höhe der wirtschaftlichen Schwelle mit entsprechend hohen Schadenssummen und der scharfen, höhenmäßig unbegrenzten Haftung der Organe und der weiten Untreuestrafbarkeit eine ausreichend wirkungsvolle Sanktion dar. “149

Tatsächlich galt die Organhaftung von Vorstand und Aufsichtsrats gegenüber der Gesellschaft bis zur Einführung des Aktiengesetzes 1937 durchaus als relevant. Demgegenüber wurde in den Neunziger Jahren des letzten Jahrhunderts die Bedeutung dieser Haftung durchweg als gering eingeschätzt. Ein Gutachter für den Leipziger Deutschen Juristentag 2000 meinte sogar, dass die Haftung jedenfalls bis zum Inkrafttreten des KonTraG „nur auf dem Papier“ gestanden habe. ${ }^{150}$ Das war auch die internationale Meinung zur Haftung von Mitgliedern des board, jedenfalls von non-executive directors. Diese Meinung hat sich teilweise noch bis heute erhalten, so wenn in einem jüngsten Aufsatz in Harvard ein preisgekrönter Aufsatz diese Meinung zum Anlass nahm, wie in Australien für eine Durchsetzung der Organhaftung durch die BaFin zu plädieren, ${ }^{151}$ eine Forderung, der allerdings der Deutsche Juristentag schon vorher eine Absage erteilt hatte. ${ }^{152}$ Inwieweit diese Meinung früher tatsächlich zutraf, müsste noch näher untersucht werden. Das war jedenfalls nicht für den Genossenschaftsbereich der Fall, und auch die Dunkelziffer aufgrund von internen Vergleichen und Schiedsgerichtsverfahren, die erst ein Blick in die internen Statistiken der D\&O-Versicherer erschloss, blieb außer Acht. ${ }^{153}$ Immerhin könnte die Haftung jedenfalls des Aufsichtsrats während der langen Periode des rheinischen Kapitalismus tatsächlich deshalb keine größere Rolle gespielt haben, weil man sich in der vernetzten Wirtschaft immer wieder begegnete und Differenzen anders regelte. Wie dem auch sei, all das hat sich aber mittlerweile deutlich geändert. ${ }^{154}$ Zwischen 1986 und 1995 gibt es 113 einschlägige Entscheidungen, zwischen 1986 und 1995206 und zwischen 1996 und 2008 165. Vor allem sind die geltend gemachten Schadensersatzsummen bis heute drastisch gestiegen. Die Gründe für diese Entwicklung liegen im Zusammenspiel der ARAG/Garmenbeck-Entscheidung des Bundesgerichtshofes 1997, der Aufsichtsratsreform ab dem KonTraG 1998, der Finanzkrise ab 2007 und dem in ihrer Folge sprunghaft angestiegenen Klima des Misstrauens gegen Banken und Aufsichtsräte. ${ }^{155}$ Auch die Ver-

149 RegE ARUG II Begründung S. 95/96.

150 Nachweise bei Норт/Roth, aaO (Fn. 1), $\$ 116$ AktG Rdn. 16.

151 Thaten, European Business Organization Law Review 19 (2018) 275: “a defunct system of director liability in Germany".

152 70. DJT 2014, Beschluss 17; dazu Норт, FS W.-H. Roth, 2015, S. 224, 239.

153 IHLAs, Organhaftung und Haftpflichtversicherung, 1997, S. $317 \mathrm{ff}$.

154 Zum Folgenden mit umfangreichen Nachweisen Hорт/Rотн, aaO (Fn. 1), $\$ 93$ AktG Rdn. $40 \mathrm{ff}, \mathbb{1 1 6}$ AktG Rdn. 17f. Für Frankreich Cozian/Viandier/Deboissy, aaO (Fn. 51), p. 157 no 272: „Un jour vient où l'orage se déclare ...“

155 Норт, ZIP 2013, 1793, 1794. 
breitung der D\&O-Versicherung mag eine Rolle spielen trotz der Schwierigkeiten, wie oft ein Konsortium mit auch im Ausland sitzenden Versicherern zur tatsächlichen Zahlung zu bewegen; ob sie allerdings für die wachsende reale Bedeutung der Haftung in der Praxis wirklich ausschlaggebend ist, wie manche meinen, ${ }^{156}$ muss dahingestellt bleiben. Mittlerweile ist sogar zu hören, dass es schwieriger geworden ist, gute Kandidaten für den Aufsichtsrat zu gewinnen. Das Verhältnis zwischen der im Vergleich zu den Vorstandsgehältern mageren Entlohnung der Aufsichtsratsmitglieder und den rechtlichen Anforderungen, den öffentlichen Erwartungen und dem Haftungsrisiko ist aus der Balance gefallen.

Es ist hier nicht der Ort aufzulisten, in welchem Umfang die Rechtsprechung und teilweise auch die Gesetzgebung die Überwachungsaufgaben des Aufsichtsrats verschärft haben. ${ }^{157}$ Am deutlichsten wird das bei den drastisch gewachsenen Anforderungen zum Risikomanagement und zur Compliance und der sich darauf erstreckenden Überwachungsverantwortung des Aufsichtsrats. ${ }^{158}$ Board Compliance ist international zu einem akuten Reformthema geworden. ${ }^{159}$ Während hinsichtlich des verschärften Pflichtenkatalogs des Aufsichtsrats und des entsprechend höheren und breiteren Anforderungsprofils von Aufsichtsratsmitgliedern weitgehend Einigkeit besteht, geht die Diskussion um die Reichweite der ARAG/Garmenbeck-Doktrin ${ }^{160}$ und der die Berufung

156 P. Doralt/W. Doralt, aaO (Fn. 32), $₫ 14$ Rdn. 24.

157 Siehe nur Норт/Rотн, aаO (Fn. 1), $\$ 116$ AktG Rdn. 72-141 zu den Einzelfällen der Sorgfaltspflicht sowie Rdn. 142-189 zu Treuepflicht und Interessenkonflikten. Auch Habersack, aaO (Fn. 1), $\mathbb{1} 116$ AktG Rdn. 29 ff. Zuletzt die Zusammenstellung bei BinZ/Sorg, BB 2019, 387. Allgemeiner Hommelhoff, FS Marsch-Barner 2018, S. 261. Das spiegelt sich auch in dem deutlich erhöhten Zeitaufwand wider. Nach einer jüngsten Befragung von 120 deutschen und österreichischen Aufsichtsratsmitgliedern betrug er durchschnittlich für Aufsichtsratsvorsitzende 46 Tage für einfache Aufsichtsratsmitglieder 25 Tage, 7 \% der Befragungsteilnehmer gaben 100 Tage für ein einzelnes Mandat an; Boston Consulting Group, Herausforderungen der Aufsichtsratsarbeit, Ergebnisse der BDG-Aufsichtsratsbefragung 2018, abrufbar unter: https://www.bcg. com/de-de/perspectives/210600 und 210607 (letzter Zugriff: 4.6.2019), auch Kurzbericht: Pidun/Stange/WolfF, Der Aufsichtsrat 04/2019, 50.

158 Schraud, aaO (Fn. 88), S. 106 ff: Compliance im Lichte der Überwachungsaufgabe des Aufsichtsrats; KorT, FS Hopt, Bd. 1, 2010, S. 983; Kleinert, FS Baums, Bd. I, 2017, S. 669; zur Haftung bei Compliance-Verstößen im Konzern Arnold/Geiger, BB 2018, 2306.

159 Armour/Garrett/Gordon/Min, Minnesota Law Review (forthcoming), auch abrufbar unter: https://ssrn.com/abstract=3205600 (letzter Zugriff: 4.6.2019). Empirische Informationen zur Relevanz eines Kontrollgremiums auf die compliance Ternes, Der Konzern 2019, 112.

160 BGH, 21.4.1997, BGHZ 135, 244, bestätigend BGH, 18.9.2018, ZIP 2018, 2117. Aus der Sicht der Praxis z.B. Reichert, ZIP 2016, 1189; Nachweise bei Hopt/Roth, aaO

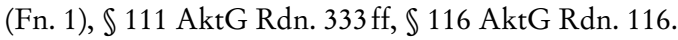


auf einen Rechtsirrtum weit zurückschneidenden Ision-Rechtsprechung des Bundesgerichtshofes ${ }^{161}$ kontrovers weiter. Dass die Aufarbeitung pflichtwidrigen Vorstandshandeln durch Aufsichtsrat und Aktionäre eine zunehmende Rolle spielt, ${ }^{162}$ ist aber nicht zu bestreiten. Dabei kommt der Beweislastverteilung in Organhaftungsprozessen eine wichtige Rolle zu. Die herkömmliche Meinung in Rechtsprechung und Schrifttum hält daran fest, dass die Beweislast das in Anspruch genommene Organmitglied trifft. Eine neuere Meinung sieht das anders, zumindest für ausgeschiedene Organmitglieder, die nach ihrem Ausscheiden keinen Zugriff mehr auf die internen Unterlagen der Gesellschaft. Der von der h.L. zugebilligte Anspruch gegen die Gesellschaft auf Herausgabe von Unterlagen ist unzureichend, denn es käme auf den Zugang zum gesamten, möglicherweise Jahre zurückliegenden Emailverkehr an, der einem ausgeschiedenen und möglicherweise bei einer Konkurrenzfirma tätigen Organmitglied offensichtlich nicht gewährt werden kann. ${ }^{163} \mathrm{Ob}$ dann der richtige Weg zu mehr Verantwortung von Organmitgliedern der ist, diese strenger in Haftung zu nehmen und diese Haftung verschärft durchzusetzen, erscheint demnach fraglich. Überzogen und nachgerade gefährlich ist jedenfalls die Rechtsprechung des Bundesgerichtshofes in Strafsachen, welche die insoweit differenzierte und richtige Organhaftungsrechtsprechung des Bundesgerichtshofs in Zivilsachen nicht beachtet. Sie wendet nämlich den Untreuetatbestand des $\$ 266 \mathrm{StGB}$ schon dann an, wenn dem Organmitglied der Haftungsfreiraum der business judgment rule nach $\ 93$ Abs. 1 Satz 2 AktG im konkreten Fall nicht zukommt. ${ }^{164}$ Der Bundesgerichtshof nimmt damit Organmitglieder schon dann strafrechtlich in Haftung, wenn sie leicht fahrlässig Fehlentscheidungen treffen und das eine oder andere der fünf Tatbestandsmerkmale der business judgment rule ${ }^{165}$ nicht vorliegt. Zivilrechtlich ist es jedoch so, dass dann, wenn die kodifizierte business

161 BGH ZIP 2011, 2097, 2099 = AG 2011, 876, 877 f (Ision); kritisch Hорт/Roth, aaO (Fn. 1), $₫ 93$ AktG Rdn. 139f, 403, \116 AktG Rdn. 275; ohne Stellungnahme, nicht

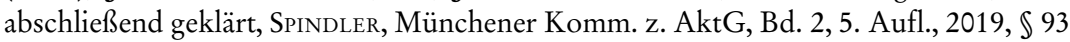
Rdn. 89. Zusammenfassend Diekmann, Das Vertrauen der Vorstandsmitglieder auf Rechtsauskünfte, 2019.

162 Holle, ZHR 182 (2018), 569.

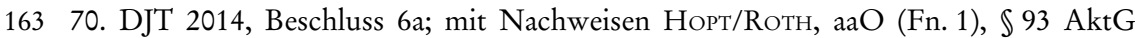
Rdn. 448 f. Näher Hopt, FS W.-H. Roth, S. 224, 232 ff. Für ausgeschiedene Organmitglieder mit einer Treupflicht der Gesellschaft zur Gewährung von Einsicht argumentierend Spindler, aaO (Fn. 1), $\mathbb{9} 93$ Rdn. 212, Fleischer, in: Spindler/Stilz, Komm. z. AktG, 4. Aufl., 2019, \93 Rdn. 224, aber das wird den heutigen technischen Möglichkeiten nicht mehr gerecht.

164 BGHSt, 12.10.2016, ZIP 2016, 2467 = NJW 2017, 578 (HSH-Nordbank), kritisch dazu Baur/Holle, ZIP 2017, 255; Kuntz DB 2017, 37, 41 f; E. Vetter/Peters, Der Konzern 2017, 269.

$165 \mathrm{Zu}$ den Einzelheiten Hорт/Rотн, aaO (Fn. 1), $₫ 93$ AktG Rdn. 71, $\$ 116$ AktG Rdn. 60; demgegenüber zur business judgment rule in den USA P. Doralt/W. Doralt, aaO (Fn. 32), $\mathbb{1} 14 \mathrm{Rdn} .84 \mathrm{ff}, 89 \mathrm{ff}$, und zur deutschen Rdn. 109. 
judgment rule ausscheidet, die Grundhaftungsnorm des $\mathbb{\$} 93$ Abs. 1 Satz 1 AktG eingreift, unter der es immer noch einen Bereich unternehmerischen Ermessens gibt ${ }^{166}$ und bei der vor allem sehr wohl zwischen leichten und schweren Verstößen unterschieden wird. Die Forderung, Banker bei Zusammenbrüchen ihrer Bank strafrechtlich zu verfolgen, ist populär, nicht nur in Deutschland, sondern auch in anderen Ländern. So wird beispielsweise in den USA und im Vereinigten Königreich unter dem Eindruck der Finanzkrise und anderer gravierender Zusammenbrüche und Skandale für mehr Strafbarkeit der directors plädiert. ${ }^{167}$ Richtig erscheint demgegenüber das Eingreifen von Strafrecht nicht schon bei leichten, sondern erst bei groben Organfehlern. ${ }^{168}$

Solange der Bundesgerichtshof bei seiner Rechtsprechung zur zivil- und insbesondere strafrechtlichen Haftung bleibt und der Gesetzgeber sich den Forderungen in der Literatur zum Investorendialog, zur vorstandsunabhängigen Information und punktuell zu den related party transactions versagt, ist der Deutsche Corporate Governance Kodex die Instanz, welche die Entwicklung selbstregulierend in die richtige Richtung weisen kann. Das hat der Kodex für den Investorendialog bereits getan, ${ }^{169}$ könnte er aber auch zur vorstandsunabhängigen Information tun, etwa dahin, dass diese, jedenfalls zunächst, ähnlich wie für Kreditinstitute ${ }^{170}$ mit den Leitern der Internen Revision und des Risikocontrolling empfohlen würde. Darüber hinaus wären eine Empfehlung zur Einrichtung eines Aufsichtsratsbüros ${ }^{171}$ sinnvoll und, wie auch im Reformentwurf 2018 geschehen, eine Empfehlung zur extern unterstützten Evaluation mindestens alle drei Jahre. ${ }^{172}$ Zuletzt bleibt nur der Appell an das Eigeninteresse des Aufsichtsrats, seine Aufgaben gut zu erfüllen, und das kann er nur, wenn er die richtigen Mitglieder hat bzw. auswählt. ${ }^{173}$

166 Норт/Roth, aaO (Fn. 1), $\$ 93$ AktG Rdn. 116ff, $\$ 116$ AktG Rdn. 62. Zutr. Fleischer, aaO (Fn. 163), $\$ 93$ AktG Rdn. 69a: $\ 93$ Abs. 1 Satz 2 AktG enthält nur eine Teilkodifikation unternehmerischer Ermessensspielräume.

167 Näher Davies/Hopt, in: Busch/Ferrarini, Governance of Financial Institutions, 2019, p. $117,6.56$ et seq.

168 Tosza, Criminal Liability of Managers in Europe, Punishing Excessive Risk, Oxford et al., 2019, p. 274 et seq.: intentional mismanagement, reckless mismanagement, negligent violation only of particular, concrete risk-preventing rules that are of grave importance for the economic system.

169 Oben III. 1. c).

170 Oben III. 2. c) zu Fn. 100.

171 Rотн, ZGR 2012, 343, 362 ff; Норт/Rотн, aаO (Fn. 1), \111 AktG Rdn. 803 f, \$ 113 AktG Rdn. 36, 39, ohne Notwendigkeit eines eigenen Budgets im Vorhinein, ebenda $\$ 111$ AktG Rdn. $530 \mathrm{ff}$; für ein eigenes Budgetrecht, das die ü.L. bisher ablehnt, SCHraud, aaO (Fn. 88), S. 164 ff, 228.

172 DCGK-E Empfehlung A.15, anders noch DCGK 5.6. Zur Evaluation bzw. Effizienzprüfung des Aufsichtsrats Норт/Rотн, aaO (Fn. 1), \$111 AktG Rdn. $221 \mathrm{ff}$.

173 Zu den Erfolgsfaktoren eines exzellenten Aufsichtsrats v Werder, DB 2017, 977; AdAMS/ AKyol/Verwijemerer, Director skill sets, Journal of Financial Economics 130 (2018) 641. 


\section{Zusammenfassung}

1. Der Aufsichtsrat ist im 19. Jahrhundert aus dem Verwaltungsrat herausgewachsen, eine strenge Trennung gab es rechtlich und praktisch nicht. Eine Geschäftsführung durch den Aufsichtsrat ist erst seit dem Aktiengesetz 1937 verboten. Der eigentliche Bedeutungswandel des Aufsichtsrats seither liegt in der unternehmerischen Mitbestimmung, im Rückzug der Banken aus den Aufsichtsräten großer Gesellschaften und in den Aufsichtsratsreformen seit den 1990er Jahren.

2. Der Bedeutungszuwachs des Aufsichtsrats erweist sich auch international. Das ein- und das zweistufige System konvergieren bei der Entscheidungsfindung, der Überwachung und der Zusammensetzung. Divergenzen (Pfadabhängigkeiten) verbleiben, so vor allem die deutsche (quasi-)paritätische Arbeitnehmermitbestimmung.

3. Der Aufsichtsrat ist heute bis zu einem gewissen Grade ein mitunternehmerisches Organ. Das zeigt sich in der allseits anerkannten prospektiven Überwachung, den dem Aufsichtsrat von Gesetzes wegen allein oder zusammen mit dem Vorstand zugewiesenen unternehmerischen Aufgaben, bei der Information des Aufsichtsrats und bei der Bedeutung und der Behandlung von Interessenkonflikten von Organen.

4. Die Diskussion über die konkrete Grenzziehung zwischen den Aufgaben und Zuständigkeitsbereichen von Vorstand und Aufsichtsrat ist hingegen in vollem Gange. Das zeigt sich beim Streit um den Investorendialog des Aufsichtsrats und um die vorstandsabhängige Information - nach der hier vertretenen Ansicht ist beides grundsätzlich möglich - und in den Meinungsverschiedenheiten um die Rolle des Aufsichtsrats bei der Umsetzung der Vorschriften der Aktionärsrechterichtlinie zu den related party transactions.

5. Der Preis für diesen Bedeutungszuwachs des Aufsichtsrats sind mehr Pflichten, namentlich was das Risikomanagement und die Compliance angeht, und eine strengere Haftung. Zur zivilrechtlichen Haftung werden kontrovers diskutiert die Reichweite der ARAG/Garmenbeck-Doktrin des Bundesgerichtshofes, seine die Berufung auf einen Rechtsirrtum weit zurückschneidende Ision-Rechtsprechung und die Beweislastumkehr zu Lasten auch des ausgeschiedenen Organmitglieds. Die Rechtsprechung des Bundesgerichtshofs in Strafsachen zur strafrechtliche Haftung der Aufsichtsratsmitglieder wegen Untreue nach $₫ 266$ StGB verkennt das Zusammenspiel von $₫ 93$ Abs. 1 Satz 2 und Satz 1 AktG und kann zur strafrechtlichen Sanktionierung schon von leicht fahrlässigem Verhaltens führen. Das ist rechtsdogmatisch nicht überzeugend, verkennt die eigentliche Rolle des Strafrechts und setzt gravierende Fehlanreize für Vorstands- und Aufsichtsratsmitglieder. 